\title{
Validation of Simple Sequence Length Polymorphism Regions of Commonly Used Mouse Strains for Marker Assisted Speed Congenics Screening
}

\author{
Channabasavaiah B. Gurumurthy, ${ }^{1}$ Poonam S. Joshi, ${ }^{1}$ Scott G. Kurz, ${ }^{1}$ Masato Ohtsuka, ${ }^{2}$ \\ Rolen M. Quadros, ${ }^{1}$ Donald W. Harms, ${ }^{1}$ and K. C. Kent Lloyd ${ }^{3}$ \\ ${ }^{1}$ Mouse Genome Engineering Core Facility, Department of Genetics, Cell Biology and Anatomy, University of Nebraska Medical Center, \\ Omaha, NE 68198-5915, USA \\ ${ }^{2}$ Department of Molecular Life Science, Division of Basic Medical Science and Molecular Medicine, Tokai University School of Medicine, \\ 143 Shimokasuya, Isehara, Kanagawa 259-1193, Japan \\ ${ }^{3}$ Mouse Biology Program, University of California, Davis, 2795 2nd Street, Davis, CA 95618, USA
}

Correspondence should be addressed to Channabasavaiah B. Gurumurthy; cgurumurthy@unmc.edu

Received 1 November 2014; Revised 30 December 2014; Accepted 10 January 2015

Academic Editor: Mohamed Salem

Copyright (c) 2015 Channabasavaiah B. Gurumurthy et al. This is an open access article distributed under the Creative Commons Attribution License, which permits unrestricted use, distribution, and reproduction in any medium, provided the original work is properly cited.

\begin{abstract}
Marker assisted speed congenics technique is commonly used to facilitate backcrossing of mouse strains in nearly half the time it normally takes otherwise. Traditionally, the technique is performed by analyzing PCR amplified regions of simple sequence length polymorphism (SSLP) markers between the recipient and donor strains: offspring with the highest number of markers showing the recipient genome across all chromosomes is chosen for the next generation. Although there are well-defined panels of SSLP makers established between certain pairs of mice strains, they are incomplete for most strains. The availability of well-established marker sets for speed congenic screens would enable the scientific community to transfer mutations across strain backgrounds. In this study, we tested the suitability of over 400 SSLP marker sets among 10 mouse strains commonly used for generating genetically engineered models. The panel of markers presented here can readily identify the specified strains and will be quite useful in marker assisted speed congenic screens. Moreover, unlike newer single nucleotide polymorphism (SNP) array methods which require sophisticated equipment, the SSLP markers panel described here only uses PCR and agarose gel electrophoresis of amplified products; therefore it can be performed in most research laboratories.
\end{abstract}

\section{Introduction}

In recent years, there has been a steady increase in the creation and use of genetically engineered mutant mice for use in biomedical research. Frequently, the genetic background of such mice allows only specific experiments and the mutations have to be transferred to different genetic background(s) to facilitate other kinds of experiments. There are many examples where genetic background is shown to influence the phenotype of a transgenic or knockout mouse line [1-12]. Traditionally however, mutant mice are generated using strains that have shown exceptional performance in terms of their suitability for production of transgenic or knockout mice lines. For example, FVB strain and BDF1 strain mice are most commonly used for transgenic mice production [13] and ES (embryonic stem) cells derived from 129 inbred strains are commonly used for knockout mice production. For technical reasons, chimeras developed in knockout mice generation will carry a mixed genetic background (e.g., 129 and B6) adding further complexity to the analysis [14]. Furthermore, ES cells derived from C57BL6/N inbred strain have been used in mouse genetic resources such as KOMP (Knock-Out Mouse Project) and EUCOMM (European Conditional Mouse Mutagenesis) [15]. Also, particular strains of mice seem to have better suitability for a specific research purpose. In addition, mixed background strains between C57BL/6J 
TABLE 1

\begin{tabular}{|c|c|c|}
\hline Strain & Vendor, stock number & Rationale \\
\hline $\mathrm{C} 57 \mathrm{BL} / 6 \mathrm{~J}$ & JaxMice, 000664 & Commonly used strain in biomedical research \\
\hline $\mathrm{FVB} / \mathrm{NJ}$ & JaxMice, 001800 & Commonly used strain for transgenic mice generation \\
\hline $129 \mathrm{X} 1 / \mathrm{SvJ}$ & JaxMice, 000691 & \multirow{2}{*}{$\begin{array}{l}\text { Strains from which highly successful germ line transmission competent ES } \\
\text { cells were derived }\end{array}$} \\
\hline $129 S 2$ & Charles River, 476 & \\
\hline DBA2/J & JaxMice, 000671 & \multirow{3}{*}{$\begin{array}{l}\text { Together with C57BL6, these strains are used as hybrid strains for } \\
\text { transgenic mouse production }\end{array}$} \\
\hline $\mathrm{CBA} / \mathrm{J}$ & JaxMice, 000656 & \\
\hline SJL/J & JaxMice, 000686 & \\
\hline $\mathrm{Balb} / \mathrm{c}$ & Charles River, 028 & Is among the top 2-3 most widely used inbred strains \\
\hline $\mathrm{C} 3 \mathrm{H} / \mathrm{HeJ}$ & Charles River 025 & \multirow{2}{*}{$\begin{array}{l}\text { Used in a variety of research areas including cancer, infectious disease, and } \\
\text { cardiovascular biology }\end{array}$} \\
\hline NOD & Charles River & \\
\hline
\end{tabular}

and DBA/2J were used for ENU mutagenesis projects because the cross between two different inbred strains (one strain (male) was used for ENU exposure and mated with another strain (female)) is useful for mapping and positional cloning of the mutated gene using genetic polymorphisms existing between them $[16,17]$. The main disadvantage of a given mutation under a particular strain background is that the strain background may limit its use for a specific research purpose. In such a situation the mutation is needed to be transferred into a strain background of choice through a process called backcrossing.

Backcrossing involves about 10 generations of successive breeding into a recipient strain of choice to achieve $99.9 \%$ congenic (genetic composition) for that strain (http://www .informatics.jax.org/silverbook/). This painstaking process consumes about 2.5 to 3 years of time, a fact that often limits its feasibility and usefulness given the pace of scientific research. In some cases, studies are published with animals after only 5 generations of backcrossing, in an attempt to compensate the time required and the need to obtain some results in the new strain [18]. A technique called "marker assisted speed congenic" used for over a decade helps in achieving congenic strain in 5 or less, unlike the usual 10 generations required in traditional backcrossing $[19,20]$. The small sequence differences between mouse strains called "microsatellite markers" served as useful tools in detecting the chromosome regions of origin in the offspring when two inbred strains of mice are bred together. To use in these assays, many microsatellite markers have been identified and characterized by various researchers between the donor and recipient strains of their choice ([21] and [22, page 6] and [23-30]). However, the information about the markers that can differentiate between commonly used strains for transgenic and knockout mice generation is not tested sufficiently and is not available readily. In this study, we tested 423 markers, $\sim 10$ to 30 per chromosome, using the genomic DNAs from 10 commonly used mice strains-particularly the strains used in transgenic and knockout research. We evaluated the markers that could be used in the agarose gel electrophoresis method which is a simple technique commonly used in most molecular biology laboratories these days. The data presented here will serve as a valuable tool for various investigators in choosing the markers useful for their speed congenic breeding.

\section{Materials and Methods}

2.1. Selection of Oligonucleotide Primers. The primers were chosen based on the following criteria: (1) evaluation and establishment of at least 6 markers per chromosome, (2) distance between the adjacent markers kept as minimum as 10 to 15 centimorgans (cM), and (3) polymorphic bands appreciable when analyzed in a $4 \%$ agarose gel electrophoresis and resolved by electrophoresis distance of up to 10 centimeters from the loading wells. All markers were chosen from the Mouse Genome Informatics (MGI) database links (http:// www.informatics.jax.org/searches/probe_report.cgi?_Refs $\mathrm{key}=22816$ and $\mathrm{ftp}$ ://ftp.informatics.jax.org/pub/data-sets/ index.html (numbers 7 and 12 in the list)).

2.2. Mice Strains. The mice were purchased from Charles River Laboratories or The Jackson Laboratory. The strains, the rationale for including these strains in the panel, and the vendors are listed in Table 1.

2.3. DNA Extraction, PCR Amplification, and Agarose Gel Electrophoresis. The DNA samples were extracted from the tail pieces of about 3-5 mm length using Gentra Puregene Tissue Kit (Cat. \# 158622). Twenty ul PCR reactions were set up using $1 \mathrm{X}$ reaction buffer containing $20 \mathrm{mM}$ Tris $\mathrm{pH} 8.4$, $50 \mathrm{mM} \mathrm{KCl}, 3 \mathrm{mM} \mathrm{MgCl}$, and 1 unit of Taq DNA polymerase (New England Biolabs, Cat. \# M0273) under the following conditions: one cycle of $95^{\circ} \mathrm{C}-2$ min followed by 35 cycles of $95^{\circ} \mathrm{C}-30 \mathrm{sec}, 55^{\circ} \mathrm{C}-30 \mathrm{sec}$, and $72^{\circ} \mathrm{C}-60 \mathrm{sec}$ and one cycle of $72^{\circ} \mathrm{C}-5$ minutes, followed by a holding temperature at $4^{\circ} \mathrm{C}$ until the samples were removed from the machine. Fifteen ul of PCR products was resolved using 4\% agarose gels for 120 to 150 minutes at 200-constant-volt electric current. The agarose was purchased from Phenix Research Products (Item Number RBA-500: Molecular Biology Grade) and the gels were prepared on $0.5 \mathrm{X}$ TAE buffer diluted from a stock of $50 \mathrm{X}$ (Fisher Scientific, Cat. \# BP1332-20). The gels contained ethidium bromide dye $(0.5 \mu \mathrm{g} / \mathrm{mL})$ to aid the visualization of PCR bands. Each panel of gel included one or more lanes of 100-base-pair molecular weight marker (New England Biolabs, Cat. \# N3231) to assess the PCR product sizes. The bromophenol blue dye-front was allowed to run for up to 10 
centimeters from the wells and the gels were imaged using BioRad Gel Doc XR system. Wherever necessary, the gels were run longer to resolve the bands.

2.4. Analysis and Interpretation of Polymorphic PCR Bands. The cropped images were imported into an Excel file for analysis and interpretation. Band sizes were assigned numbers 1 , 2,3 , or 4 to indicate their sizes compared to the rest of the bands in that set. Number 1 was assigned to the smallest sized polymorphic band, 2 to the next biggest in the group, and so on. The Excel file along with the gel images was converted into a .pdf file for generating Figure 1 .

\section{Results and Discussion}

Mutant mice created using transgenic and knockout techniques are available under certain specific backgrounds. In order to best use such mutants for a specific research purpose, they routinely need to be bred into other strain backgrounds through successive breeding of about 10 generations. A quicker way to attain highest recipient genome can be achieved by a process called speed congenic breeding in which the polymorphic markers between the recipient and donor strains are screened among offspring in each generation and the offspring with highest recipient genome is chosen as a breeder for the subsequent generation [19-21]. Although there are a few reports describing the marker sets suitable for certain pairs of strains, there are no wellestablished marker sets available across the most commonly used strains in transgenic and knockout mouse techniques. Our primary objective of this work was to test the suitability of several markers in an agarose gel electrophoresis method, a technique that uses least expensive equipment and reagents and is readily available in most molecular biology laboratories. We sought to test a large number of microsatellite markers (Tables 2 and 3 and S1 in Supplementary Material available online at http://dx.doi.org/10.1155/2015/735845) among 10 most commonly used mouse strains particularly those used in transgenic and knockout mice techniques.

We used Mouse Genome Informatics (MGI) database and short-listed the primers based on the criteria described in Section 2. MGI database lists several of the microsatellite markers identified in various inbred strains. Although the database is extensive, it is difficult to choose marker sets for speed congenic screening because it lacks the critical information such as the sizes of PCR products of various markers in different inbred strains and if the differences among strains can be identified using conventional agarose gel electrophoresis.

As per the information available on MGI database, predicted PCR band size differences among strains for some markers ranged from as few as a few base pairs to over 100 base pairs. Selection criteria for markers and the mice strain are described in Section 2. Taking C57BL6J and 129X1 strains as a comparison pair, for example, we chose markers with differences of $30 \mathrm{bp}$ and above (as per MGI database), a range that can be easily resolved using about $2 \%$ agarose gels. We aimed to choose markers in this range for all the chromosomal locations with an interval of 5 to $15 \mathrm{cM}$. However, we were unable to find suitable markers in some regions with this criterion. In such locations, we tested markers with as less as 8 to $12 \mathrm{bp}$ size difference. Such small differences can be best resolved using polyacrylamide gel electrophoresis (PAGE). However, in a typical speed congenic screening that involves analysis of about 100 markers for each sample, applying PAGE method for screening becomes quite laborious. We used $4 \%$ agarose gels to resolve makers even with very small size differences; this seemed to be sufficient to resolve the bands when they were run about 8 to 10 centimeters from the loading well. In order to keep the assay conditions uniform we used $4 \%$ agarose gel for all the markers tested.

Table 2 shows the list of the markers tested and found polymorphic in at least one of the 10 strains analyzed. We tested a total of 423 markers of which we present useful data for 195 markers. The gel images and interpretation of polymorphisms are tabulated in Figure 1. The gel images indicate that there were readily appreciable size differences for most markers whereas some markers showed very narrow size difference between some strains. In general, sizes of the majority of the markers matched the information on MGI database but there were some discrepancies noticed. The agarose gel electrophoresis using $4 \%$ gels could be useful in detecting differences among the markers in different strains.

The data presented in Figure 1 indicate that some markers could readily distinguish several strains from each other. We initially aimed to identify good panel of markers for every $10 \mathrm{cM}$. Although we found adjacent markers as close as $10 \mathrm{cM}$ in most locations, we failed to achieve this in some regions. We screened several available markers in such regions and were unable to find markers that matched the criteria set forth in our assay. It should be noted that there were markers that differed very minutely among some strains. If such markers are chosen in an actual speed congenic screening, we recommend including samples from both donor and recipient strains and an equimolar mixture of the two in the panel in order to ensure the proper banding pattern of offspring. The gel images and the interpretation of polymorphism provided in Figure 1 will serve as a comprehensive tool for a researcher who wishes to undertake congenic breeding in a pair of strains from the list.

The data from the markers that did not meet our criteria fell into one of the following categories: it (1) yielded the same size bands in all the strains, (2) failed to amplify a product in some strains, (3) amplified multiple PCR products, or (4) did not yield a reliable PCR product in most/all of the strains. Category 1 means that the bands were not resolvable by $4 \%$ agarose gels. We presume that such markers would be $<6$ base pairs different from one another [31] or they do not differ in size at all. Category 2 would probably mean that the primers did not have perfect binding sites in those strains where there was no PCR product. Category 3 makers may need further optimization of PCR conditions. However, one of our aims was to keep the conditions uniform to all the markers in order to keep the assay simple: it makes the assay cumbersome if PCR conditions have to be varied among different marker sets. It should be noted that the category 4 markers (that did 


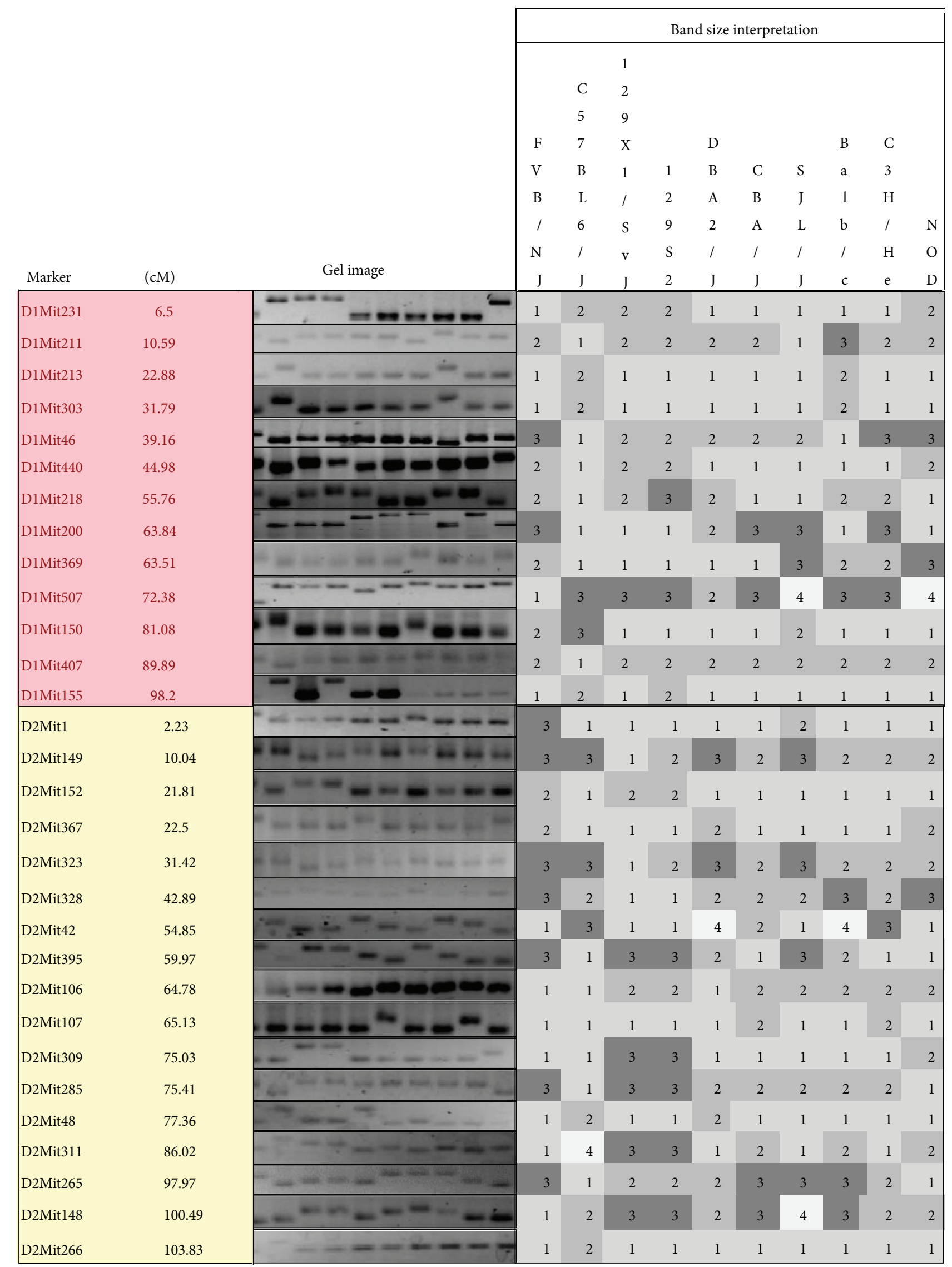

Figure 1: Continued. 


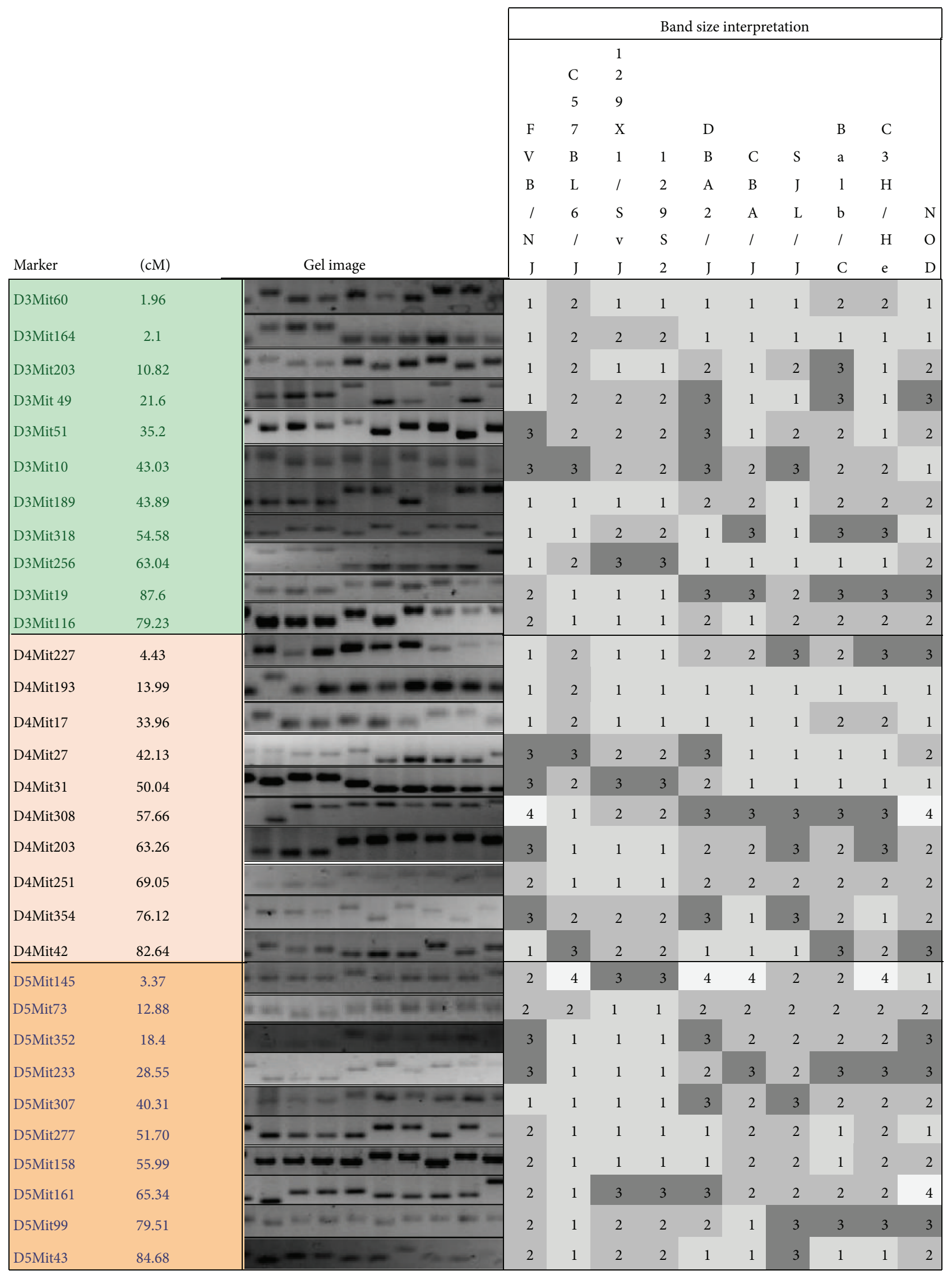

Figure 1: Continued. 


\begin{tabular}{|c|c|c|c|c|c|c|c|c|c|c|c|c|}
\hline & & & & & & $\mathrm{d}$ si & inte & reta & & & & \\
\hline & & & & & 1 & & & & & & & \\
\hline & & & & $\mathrm{C}$ & 2 & & & & & & & \\
\hline & & & & 5 & 9 & & & & & & & \\
\hline & & & $\mathrm{F}$ & 7 & $\mathrm{X}$ & & $\mathrm{D}$ & & & $\mathrm{B}$ & $\mathrm{C}$ & \\
\hline & & & $\mathrm{V}$ & $\mathrm{B}$ & 1 & 1 & $\mathrm{~B}$ & $\mathrm{C}$ & $S$ & $\mathrm{a}$ & 3 & \\
\hline & & & B & $\mathrm{L}$ & l & 2 & A & $\mathrm{B}$ & $\mathrm{J}$ & 1 & $\mathrm{H}$ & \\
\hline & & & l & 6 & S & 9 & 2 & A & $\mathrm{L}$ & $\mathrm{b}$ & / & $\mathrm{N}$ \\
\hline & & & $\mathrm{N}$ & l & $\mathrm{v}$ & S & l & l & l & l & $\mathrm{H}$ & $\mathrm{O}$ \\
\hline Marker & $(\mathrm{cM})$ & Gel image & $\mathrm{J}$ & $\mathrm{J}$ & $\mathrm{J}$ & 2 & $\mathrm{~J}$ & $\mathrm{~J}$ & $\mathrm{~J}$ & $\mathrm{C}$ & $\mathrm{e}$ & $\mathrm{D}$ \\
\hline D6Mit138 & 1.81 & $-2=-2+-$ & 3 & 1 & 3 & 2 & 2 & 2 & 3 & 3 & 4 & 3 \\
\hline D6Mit296 & 2.25 & $-1-2-2-1=0$ & 1 & 1 & 1 & 1 & 2 & 1 & 1 & 1 & 1 & 2 \\
\hline D6Mit116 & 11.5 & 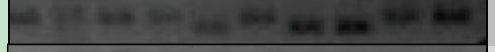 & 2 & 2 & 2 & 2 & 1 & 2 & 1 & 1 & 2 & 2 \\
\hline D6Mit274 & 23.70 & $-3 \cdot 2-1-1-1$ & 1 & 2 & 2 & 2 & 1 & 1 & 1 & 1 & 1 & 1 \\
\hline D6Mit8 & 35.94 & $------1-1$ & 3 & 1 & 2 & 2 & 1 & 1 & 3 & 3 & 2 & 3 \\
\hline D6Mit100 & 41.03 & $-5---\infty--^{-}$ & 2 & 1 & 1 & 1 & 1 & 1 & 1 & 1 & 1 & 2 \\
\hline D6Mit36 & 48.93 & $--\cdots-\cdots,-\cdots$ & 1 & 3 & 2 & 2 & 3 & 2 & 1 & 2 & 2 & 3 \\
\hline D6Mit10 & 52.75 & 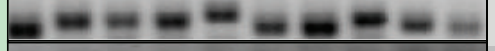 & 1 & 2 & 2 & 2 & 3 & 1 & 1 & 2 & 1 & 1 \\
\hline D6Mit333 & 60.55 & 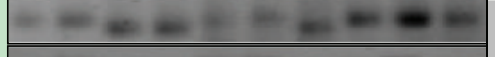 & 2 & 2 & 1 & 1 & 2 & 2 & 1 & 2 & 2 & 2 \\
\hline D6Mit302 & 67.66 & 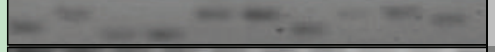 & 2 & 3 & 1 & 1 & 3 & 3 & 2 & 3 & 3 & 2 \\
\hline D6Mit14 & 77.64 & $-------1-0$ & 3 & 2 & 2 & 2 & 1 & 2 & 1 & 2 & 1 & 3 \\
\hline D7Mit21 & 1.91 & $5-m-m-m-m$ & 1 & 1 & 2 & 2 & 2 & 2 & 1 & 1 & 2 & 1 \\
\hline D7Mit152 & 2.71 & 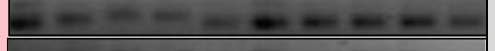 & 1 & 2 & 3 & 3 & 1 & 1 & 1 & 1 & 1 & 1 \\
\hline D7Mit57 & 9.94 & - & 1 & 2 & 2 & 2 & 1 & 2 & 2 & 2 & 2 & 2 \\
\hline D7Mit267 & 17.09 & $-\quad-2---$ & 3 & 2 & 3 & 3 & 1 & 2 & 1 & 1 & 3 & 2 \\
\hline D7Mit158 & 29.82 & 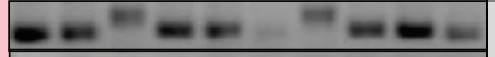 & 1 & 1 & 2 & 1 & 1 & 1 & 2 & 1 & 1 & 1 \\
\hline D7Mit317 & 41.50 & \begin{tabular}{|l|l|} 
\\
\end{tabular} & 1 & 4 & 2 & 2 & 3 & 2 & 2 & 3 & 3 & 2 \\
\hline D7Mit194 & 44.83 & 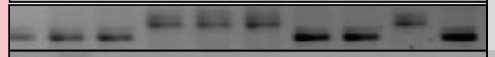 & 1 & 1 & 1 & 2 & 2 & 2 & 1 & 1 & 2 & 1 \\
\hline D7Mit220 & 55.69 & $--1---1--1$ & 2 & 3 & 2 & 2 & 2 & 2 & 1 & 2 & 2 & 2 \\
\hline D7Mit101 & 69.01 & $---\cdots---\cdots$ & 2 & 2 & 2 & 2 & 2 & 2 & 1 & 1 & 2 & 3 \\
\hline D7Mit223 & 88.85 & 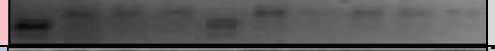 & 1 & 1 & 2 & 2 & 1 & 1 & 1 & 1 & 1 & 1 \\
\hline D8Mit143 & 11.59 & 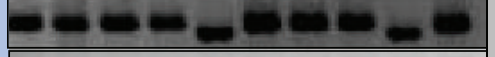 & 2 & 2 & 2 & 2 & 1 & 2 & 2 & 2 & 1 & 2 \\
\hline D8Mit63 & 19.02 & $-\ldots-\cdots-\cdots-\cdots$ & 2 & 1 & 2 & 2 & 2 & 3 & 3 & 3 & 3 & 3 \\
\hline D8Mit178 & 34.43 & 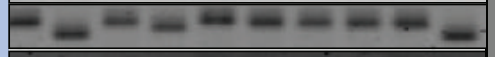 & 3 & 1 & 3 & 2 & 3 & 3 & 3 & 3 & 3 & 1 \\
\hline D8Mit45 & 42.16 & 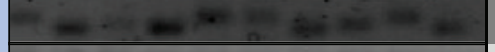 & 3 & 1 & 2 & 1 & 3 & 3 & 1 & 2 & 3 & 2 \\
\hline D8Mit80 & 43.06 & $2-2-2-2=$ & 1 & 1 & 2 & 2 & 1 & 1 & 1 & 2 & 3 & 2 \\
\hline D8Mit211 & 52.0 & 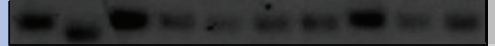 & 2 & 1 & 2 & 2 & 2 & 2 & 2 & 2 & 2 & 2 \\
\hline D8Mit215 & 62.63 & 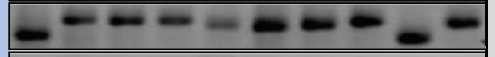 & 1 & 3 & 3 & 3 & 2 & 2 & 2 & 3 & 1 & 2 \\
\hline D8Mit56 & 76.14 & 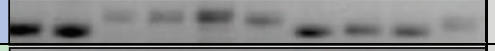 & 1 & 1 & 3 & 3 & 3 & 3 & 1 & 1 & 1 & 2 \\
\hline D9Mit249 & 2.46 & $+-a_{-1}-\ldots-1$ & 1 & 1 & 2 & 2 & 1 & 1 & 1 & 1 & 1 & 1 \\
\hline D9Mit250 & 2.46 & $\begin{array}{|cc|}-\infty-\infty & -\infty \\
\end{array}$ & 1 & 1 & 2 & 2 & 1 & 1 & 1 & 1 & 1 & 1 \\
\hline D9Mit89 & 14.79 & $-2-2=0$ & 3 & 2 & 2 & 2 & 2 & 1 & 3 & 2 & 2 & 2 \\
\hline D9Mit285 & 21.42 & $--1--1--1$ & 1 & 3 & 2 & 2 & 4 & 1 & 2 & 1 & 1 & 1 \\
\hline D9Mit336 & 35.39 & $2-z^{2}-2-1-2$ & 1 & 2 & 2 & 2 & 2 & 3 & 1 & 2 & 2 & 3 \\
\hline D9Mit8 & 42.65 & 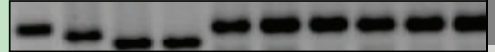 & 3 & 2 & 1 & 1 & 3 & 3 & 3 & 3 & 3 & 3 \\
\hline D9Mit347 & 55.11 & $-2--m--m$ & 1 & 3 & 3 & 3 & 4 & 4 & 2 & 3 & 3 & 3 \\
\hline D9Mit18 & 71.49 & 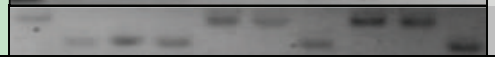 & 2 & 1 & 1 & 1 & 2 & 2 & 1 & 2 & 2 & 1 \\
\hline
\end{tabular}

Figure 1: Continued. 


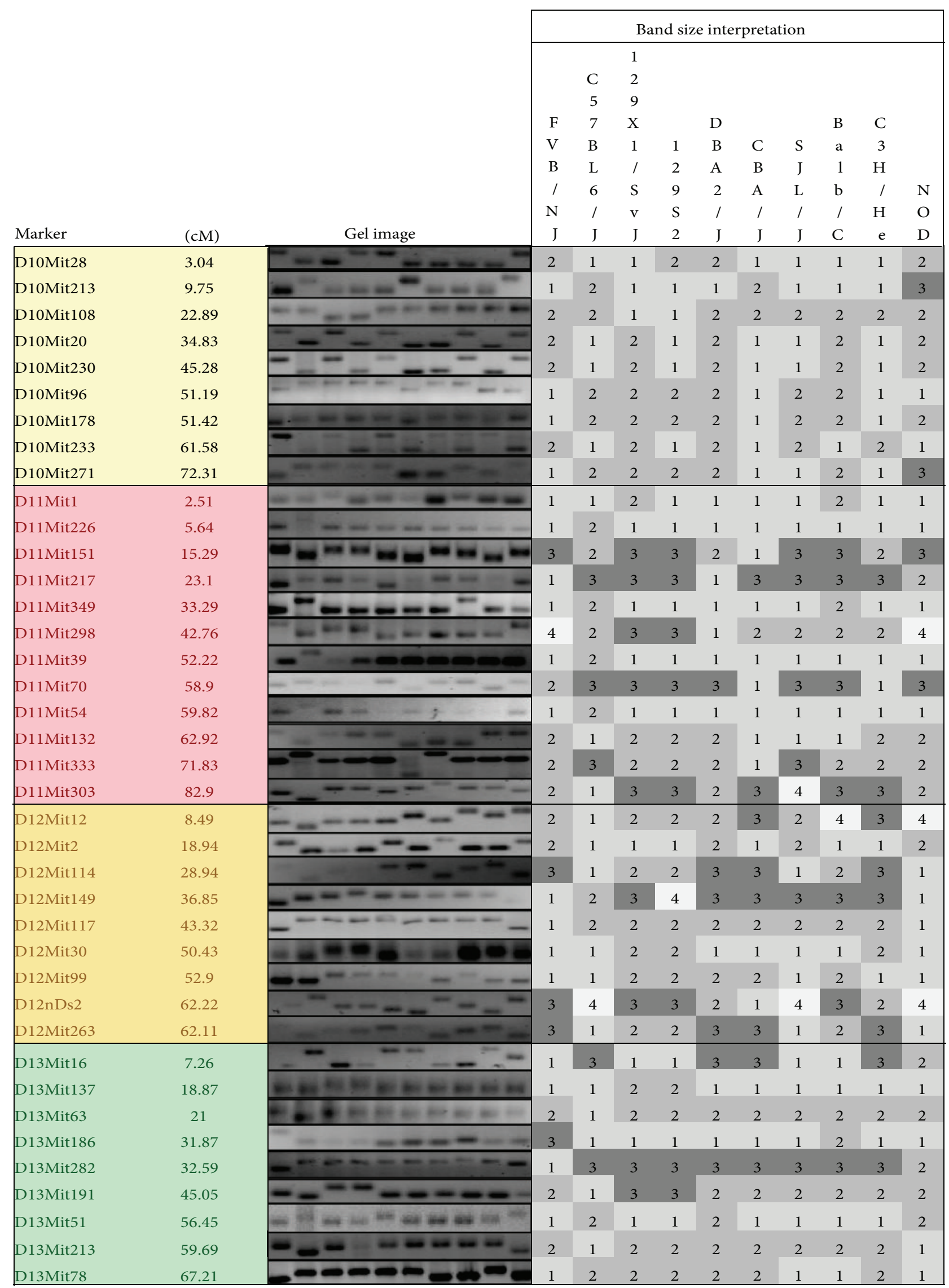

Figure 1: Continued. 


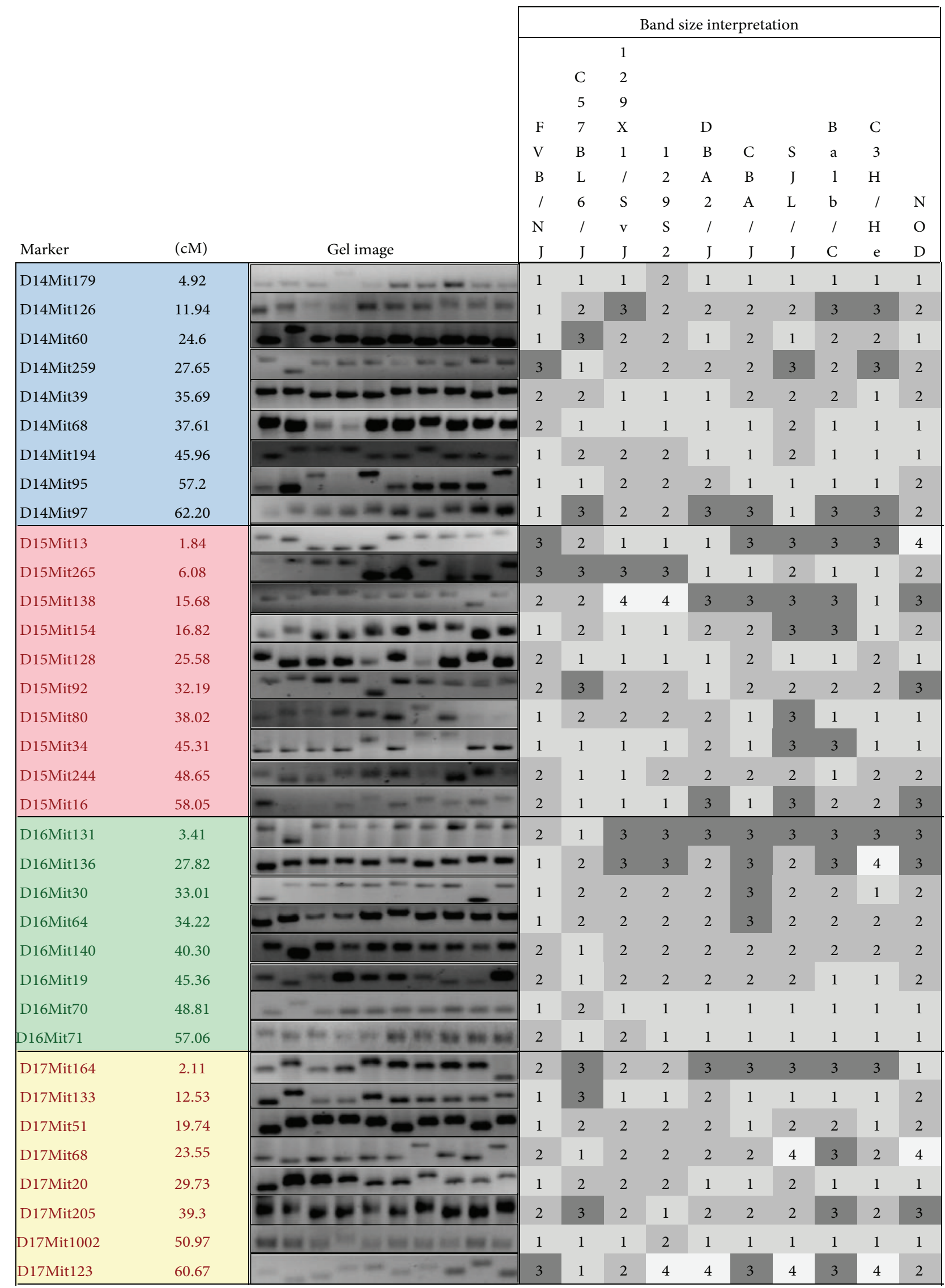

FIgure 1: Continued. 


\begin{tabular}{|c|c|c|c|c|c|c|c|c|c|c|c|c|}
\hline & & & & & & & nd & int & reta & & & \\
\hline & & & & & 1 & & & & & & & \\
\hline & & & & $\mathrm{C}$ & 2 & & & & & & & \\
\hline & & & & 5 & 9 & & & & & & & \\
\hline & & & $\mathrm{F}$ & 7 & $\mathrm{X}$ & & D & & & B & $\mathrm{C}$ & \\
\hline & & & $\mathrm{V}$ & B & 1 & 1 & B & $\mathrm{C}$ & S & $\mathrm{a}$ & 3 & \\
\hline & & & B & $\mathrm{L}$ & l & 2 & A & B & $\mathrm{J}$ & 1 & $\mathrm{H}$ & \\
\hline & & & I & 6 & S & 9 & 2 & A & $\mathrm{L}$ & $\mathrm{b}$ & I & $\mathrm{N}$ \\
\hline & & & $\mathrm{N}$ & I & $\mathrm{v}$ & S & I & I & I & I & $\mathrm{H}$ & $\mathrm{O}$ \\
\hline Marker & $(\mathrm{cM})$ & Gel image & $\mathrm{J}$ & $\mathrm{J}$ & $\mathrm{J}$ & 2 & $\mathrm{~J}$ & $\mathrm{~J}$ & $\mathrm{~J}$ & $\mathrm{C}$ & $\mathrm{e}$ & D \\
\hline D18Mit222 & 8.08 & $-F-m-1-1-$ & 2 & 2 & 2 & 2 & 2 & 1 & 2 & 1 & 1 & 1 \\
\hline D18Mit226 & 18.18 & $\mid$ & 2 & 1 & 2 & 1 & 1 & 1 & 2 & 2 & 2 & 2 \\
\hline D18Mit177 & 21.39 & 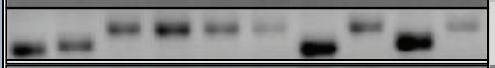 & 1 & 2 & 3 & 3 & 3 & 3 & 1 & 3 & 2 & 3 \\
\hline D18Mit91 & 29.67 & 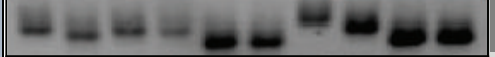 & 3 & 2 & 3 & 3 & 1 & 1 & 4 & 3 & 2 & 2 \\
\hline D18Mit124 & 32.15 & 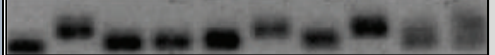 & 1 & 3 & 2 & 2 & 2 & 3 & 2 & 3 & 2 & 2 \\
\hline D18Mit184 & 39.70 & 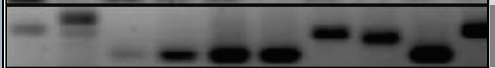 & 3 & 4 & 1 & 1 & 1 & 1 & 3 & 2 & 1 & 3 \\
\hline D18Mit186 & 45.63 & $\mid-\omega^{-\infty}--\ldots$ & 1 & 2 & 1 & 3 & 3 & 3 & 3 & 2 & 3 & 2 \\
\hline D18Mit7 & 51.92 & 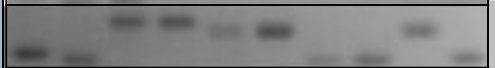 & 2 & 1 & 4 & 4 & 3 & 3 & 1 & 1 & 3 & 1 \\
\hline D18Mit129 & 53.28 & 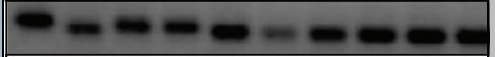 & 2 & 1 & 2 & 2 & 1 & 1 & 1 & 1 & 1 & 1 \\
\hline D19Mit68 & 3.38 & 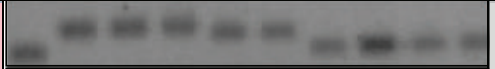 & 1 & 2 & 2 & 2 & 2 & 2 & 1 & 1 & 1 & 1 \\
\hline D19Mit60 & 13.9 & $\sec \sec$ & 1 & 2 & 2 & 2 & 2 & 2 & 1 & 2 & 2 & 1 \\
\hline D19Mit45 & 16.14 & 6- & 3 & 2 & 2 & 2 & 1 & 1 & 3 & 2 & 1 & 1 \\
\hline D19Mit30 & 21.34 & 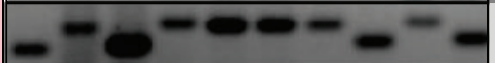 & 1 & 3 & 1 & 3 & 3 & 3 & 3 & 2 & 3 & 2 \\
\hline D19Mit88 & 32.23 & 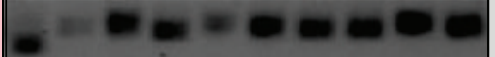 & 1 & 2 & 2 & 2 & 2 & 2 & 2 & 2 & 2 & 2 \\
\hline D19Mit19 & 34.08 & 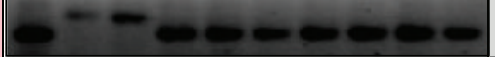 & 1 & 2 & 2 & 1 & 1 & 1 & 1 & 1 & 1 & 1 \\
\hline D19Mit11 & 36.26 & | & 3 & 1 & 1 & 4 & 2 & 4 & 4 & 3 & 4 & 3 \\
\hline D19Mit91 & 40.53 & 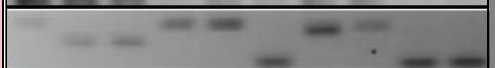 & 3 & 2 & 2 & 3 & 3 & 1 & 3 & 3 & 1 & 1 \\
\hline D19Mit1 & 50.32 & $6 \mathrm{sen}$ & 2 & 1 & 1 & 2 & 2 & 2 & 2 & 2 & 2 & 2 \\
\hline D19Mit71 & 56.28 & 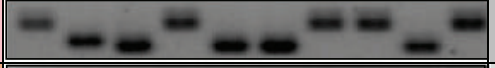 & 2 & 1 & 1 & 2 & 1 & 1 & 2 & 2 & 1 & 2 \\
\hline DXMit136 & 4.23 & 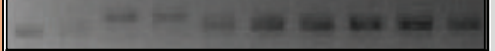 & 1 & 1 & 2 & 2 & 1 & 1 & 1 & 1 & 1 & 1 \\
\hline DXMit48 & 25.51 & 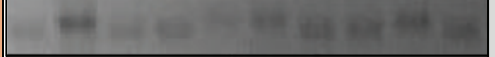 & 1 & 2 & 1 & 1 & 2 & 2 & 1 & 1 & 2 & 1 \\
\hline DXMit62 & 34.6 & 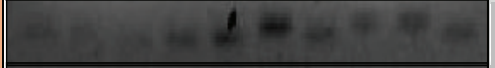 & 2 & 2 & 1 & 1 & 1 & 2 & 1 & 2 & 2 & 1 \\
\hline DXMit110 & 35.53 & 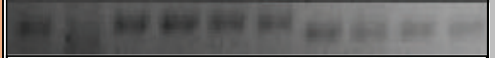 & 2 & 1 & 2 & 2 & 2 & 2 & 1 & 1 & 1 & 1 \\
\hline DXMit170 & 45.87 & 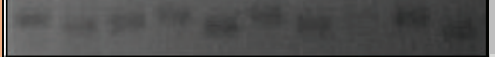 & 2 & 1 & 2 & 3 & 1 & 3 & 1 & 3 & 2 & 1 \\
\hline DXMit179 & 53.17 & & 1 & 1 & 1 & 1 & 1 & 1 & 1 & 1 & 1 & 2 \\
\hline DXMit79 & 68.46 & 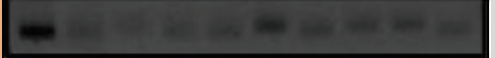 & 1 & 1 & 2 & 1 & 1 & 2 & 1 & 2 & 2 & 1 \\
\hline DXMit153 & 73.26 & 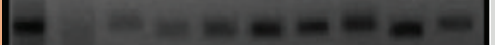 & 1 & 1 & 2 & 1 & 1 & 1 & 1 & 2 & 1 & 2 \\
\hline
\end{tabular}

FIGURE 1: Agarose gel images of PCR bands and their interpretation. Bands were assigned numbers 1, 2, 3, or 4 to indicate their sizes compared to the rest of the bands in that set. Number 1 was assigned to the smallest sized polymorphic band. 2, 3, and 4 represent the successive bigger sized bands. 
TABLE 2: Primer sequences used for PCR amplification of SSLP markers.

\begin{tabular}{|c|c|c|c|c|c|}
\hline Marker & $\mathrm{cM}$ & Primer F & Primer R & Product size & Lab code \\
\hline D1Mit231 & 6.5 & ACCCACAATTGCCTGTGG & GTCTTTGCAAGCCACCAAAT & 267 & $1-1$ \\
\hline D1Mit211 & 10.59 & GTTATTCATCAAAATACAGATGGCC & TCTGCTGCTAAGTAGAATGAATGC & 135 & $1-2 B$ \\
\hline D1Mit213 & 22.88 & TTCTTAGAAGTGATAAAAGTTTCAGCA & AAAATTCCAGAATTCTCACTACGG & 108 & $1-2$ \\
\hline D1Mit303 & 31.79 & GGTTTCTATTTCGGTTCTCGG & TCTGTGCTGCAAAACAGAGG & 128 & $1-3$ \\
\hline D1Mit46 & 39.16 & AGTCAGTCAGGGCTACATGATG & CACGGGTGCTCTATTTGGAA & 253 & $1-4$ \\
\hline D1Mit440 & 44.98 & TCCACACAAGGTGTCCTCTG & GCTCAGGTGACCTCCAAAAC & 114 & $1-5 B$ \\
\hline D1Mit218 & 55.76 & TGCAAATGTTACTTTAGTCTCTAGTGG & AGTTTTGGTGAGTGATCTTATCCC & 145 & $1-218$ \\
\hline D1Mit369 & 63.51 & ACTTGTTTGTTGCTGAGGTTCA & GCTTATGAACCCACССТCAA & 141 & $1-5 \mathrm{Q}$ \\
\hline D1Mit200 & 63.84 & GCCATGTTCATGTACATAGGTAGG & ATGGATGGATGGTTTTCCTG & 199 & $1-200$ \\
\hline D1Mit507 & 72.38 & GTTGGAAAGACTGTTTACAATTCG & GTTCCAGCCTTTGCCCTTAC & 115 & $1-6$ \\
\hline D1Mit150 & 81.08 & CTGGTGTCCACACACAGGTC & TGATTGCAATATACCAGGTTTCC & 138 & $1-150$ \\
\hline D1Mit407 & 89.89 & GAGAACAACCAGCCACCAAT & ATATTTGCTTTGAAGTTACTTTGTGTG & 119 & $1-407$ \\
\hline D1Mit155 & 98.2 & ATGCATGCATGCACACGT & ACCGTGAAATGTTCACCCAT & 252 & $1-7$ \\
\hline D2Mit1 & 2.23 & CTTTTTCGTATGTGGTGGGG & AACATTGGGCCTCTATGCAC & 123 & $2-1$ \\
\hline D2Mit149 & 10.04 & ATATCATATAGTAGAGAAAGCGTGCTG & TCATTAGACTTGGAAAAAAGTTTGC & 199 & $2-1 \mathrm{P}$ \\
\hline D2Mit152 & 21.81 & CACAGATCTTGTAAGACCACGTG & TGCCATGAGTGTGGGACTAA & 109 & $2-2$ \\
\hline D2Mit367 & 22.5 & GCCTGTGCTAAAAAAGAGGTG & GCCCTGAGAACTACССТССТ & 149 & $2-367$ \\
\hline D2Mit323 & 31.42 & AGAATCCTAAGTGGTGGTTAGAGG & ACCCAAAGTTGTCTTTAAGTACACA & 125 & $2-2 \mathrm{P}$ \\
\hline D2Mit328 & 42.89 & CTTTCAATGTTCCGGCATG & AAGACTTGCTTTCATTAGACCACA & 235 & $2-3$ \\
\hline D2Mit42 & 54.85 & ATTACTGGGCAGGAACATTTG & GCCAAACTTCCAGACTCCTC & 132 & $2-3 B$ \\
\hline D2Mit395 & 59.97 & AGGTCAGCCTGGACTATATGG & AGCATCCATGGGATAATGGT & 125 & $2-4$ \\
\hline D2Mit106 & 64.78 & GAGGGTTGCCAAAGAGACTG & CACCTCAGGGGAACATTGTG & 150 & $2-4 \mathrm{P}$ \\
\hline D2Mit107 & 65.13 & GGGAGTGAAGCCAGCATAAG & AACTGACTGAGTTTCAAAGTGCC & 119 & $2-107$ \\
\hline D2Mit309 & 75.03 & ACAAATGCCACTCTCACATCC & TATTTCTCAGAGTCACTAGGAGTGATG & 119 & $2-309$ \\
\hline D2Mit285 & 75.41 & TCAATCCCTGTCTGTGGTAGG & TATGACACTTACAAGGTTTTTGGTG & 141 & $2-5$ \\
\hline D2Mit48 & 77.36 & GCTCTGCAGAAGATGCTGC & GCTGAGACGCAGAGTCGC & 130 & $2-5 B$ \\
\hline D2Mit311 & 86.02 & ACAGGCAGCCTTCCCTTC & TCTGTCCCGCTTCTGTTTCT & 126 & $2-5 \mathrm{D}$ \\
\hline D2Mit265 & 97.97 & AATAATAATCAAGGTTGTCATTGAACC & TAGTCAAAATTCTTTTGTGTGTTGC & 105 & $2-6 \mathrm{P}$ \\
\hline D2Mit266 & 103.83 & GGATCTATGCTCCATTTTAATTGC & TCATCTTCTGGTTTCAACATGG & 127 & $2-6$ \\
\hline D2Mit148 & 100.49 & GTTCTCTGATCTACGGGCATG & TTCACTTCTACAAGTTCTACAAGTTCC & 115 & $2-148$ \\
\hline D3Mit60 & 1.96 & GACATCCTGGGCAACATTG & GGTGTTGTTTGCTGTTGCTG & 170 & $3-1$ \\
\hline D3Mit164 & 2.01 & GCTCCTGGGAAAGGAAGAAT & GATACTTGGGGTTGTGCATACA & 135 & $3-164$ \\
\hline D3Mit203 & 10.82 & CTGAATCCTTATGTCCACTGAGG & GGGCACCTGCATTCATGT & 150 & $3-2$ \\
\hline D3Mit51 & 35.2 & GGCACTGATAGCAGGCCTAG & TCTCTTCTGGTATTTCCTTCCG & 248 & $3-3$ \\
\hline D3Mit 49 & 39.02 & СTTTTCTCGCCCCACTTTC & TCCTTTTAGTTTTTGATCCTCTGG & 132 & $3-49$ \\
\hline D3Mit10 F & 43.03 & CTGGCTTGGTGGAAGTCCT & CCTAAGCCAGCTACCACCAC & 142 & 310 \\
\hline D3Mit189 & 43.89 & GTTACCACCCAGAGAAAAGGC & TACTCCTCGCTGCTTCCCTA & 133 & $3-189$ \\
\hline D3Mit318 & 54.58 & CTCATTCCTTCTGAGCAATGG & TATGGGATATGCTTTTCATAAAAGG & 148 & $3-318$ \\
\hline D3Mit256 & 63.04 & TACATTGCTTTTTGCTTTGAGTG & GTCGAATGTTATCAGAATTTGCA & 125 & $3-4$ \\
\hline D3Mit116 & 79.23 & TCACTGCCCATCTTTGTAACC & CCCAGAGACCCGGAATAGAA & 259 & $3-116$ \\
\hline D3Mit19 & 87.6 & CAGCCAGAGAGGAGCTGTCT & GAACATTGGGGTGTTTGCTT & 159 & $3-5$ \\
\hline D4Mit227 & 4.43 & CTCAGACATGATTTTTTCCAAGG & GCAGTTAAACTGTACTTTCTGTAAACA & 181 & $4-1$ \\
\hline D4Mit193 & 13.99 & TATTTTAATTTTAGCCCATCAGGG & AAAGACATACAATTGATCCACAGG & 136 & $4-1 \mathrm{~B}$ \\
\hline D4Mit17 & 33.96 & TGGCCAACCTCTGTGCTTCC & ACAGTTGTCCTCTGACATCC & 147 & $4-2$ \\
\hline D4Mit27 & 42.13 & GCACGGTAGTTTTTCCAGGA & TGGTGGGCAGGCAATAGT & 150 & $4-2 \mathrm{~B}$ \\
\hline D4Mit31 & 50.04 & ACGAGTTGTCCTCTGATCAACA & AGCCAGAGCAAACACCAACT & 121 & $4-2 \mathrm{C}$ \\
\hline D4Mit308 & 57.66 & TATGGATCCACTCTCCAGAAA & CAAAGTCTCCTCCAAGGCTG & 88 & $4-3$ \\
\hline D4Mit203 & 63.26 & GAATTCTTCCTGGGCCTTTC & CAAGAGCCCAGGTGTGGTAT & 105 & $4-3 \mathrm{~B}$ \\
\hline D4Mit251 & 69.05 & AAAAATCGTTCTTTGACTTCTACATG & TTTAAAAGGGTTTCTTTATCCTGTG & 114 & $4-4$ \\
\hline D4Mit354 & 76.12 & TTGATCTGTCGTGGATTCCA & AGACAGACACATAGACACAGACATAGG & 111 & $4-4 \mathrm{~B}$ \\
\hline D4Mit42 & 82.64 & CATGTTTGCCACCCTGAAAC & ССTCACTTAGGCAGGTGACTC & 100 & $4-5$ \\
\hline
\end{tabular}


TABLE 2: Continued.

\begin{tabular}{|c|c|c|c|c|c|}
\hline Marker & $\mathrm{cM}$ & Primer $\mathrm{F}$ & Primer R & Product size & Lab code \\
\hline D5Mit145 & 3.37 & TATCAGCAATACAGACTCAGTAGGC & TGCCCCTTAAATTCATGGTC & 150 & $5-1$ \\
\hline D5Mit73 & 12.88 & GTTTGAGAGGTCCTGAAAGCA & TTTCCATTTACATACATTTGTGCA & 113 & $5-1 \mathrm{~B}$ \\
\hline D5Mit352 & 18.4 & CCCAGAGCCCACATCAAG & TAGGTGGGTGTGTCTCTCCC & 110 & $5-2$ \\
\hline D5Mit233 & 28.55 & TCСССТCTGATCTCСТCAGA & ССТCСТAGAATACAATTCAATGTGG & 147 & $5-3$ \\
\hline D5Mit307 & 40.31 & AGTGGCATAACTTCATTCAATAATG & GGAATCAAGTTGTTTTTTAAATTTACC & 120 & $5-3 \mathrm{P}$ \\
\hline D5Mit277 & 51.7 & GTGTGTTTGTGCATGGGTATG & ACCATCGGGAAAAAATGTAGC & 123 & $5-4 \mathrm{~B}$ \\
\hline D5Mit161 & 65.34 & CACACGCATAGTCTTGTGGG & GCATGTTCAACTGTGCTTTCA & 119 & $5-5 B$ \\
\hline D5Mit99 & 79.51 & CAGAAAAGAGAAAACGGAGGG & TTCCTGCTGCCTGAAGTTTT & 200 & $5-5 \mathrm{C}$ \\
\hline D6Mit138 & 1.81 & GCTCTTATTAATGAAGAAGAAGGAGG & CAAAGAAAGCATTTCAAGACTGC & 135 & 6-1 \\
\hline D6Mit296 & 2.25 & TCGGGCATCTTTATTTTTGC & TAGTGCAGCACACCCCCT & 100 & 6-296 \\
\hline D6Mit116 & 11.5 & ACATTTCTTTGTGAGGTTCCTTG & CAGGTTTTTTGAAAGACACTCTTG & 122 & $6-1 \mathrm{~B}$ \\
\hline D6Mit274 & 23.7 & GCAATGCCAAAATGTTCAAA & ТССТТСТССАТТТАСАСТТАСАAСА & 113 & $6-1 \mathrm{D}$ \\
\hline D6Mit8 & 35.94 & TGCACAGCAGCTCATTCTCT & GGAAGGAAGGAGTGGGGTAG & 163 & $6-2 \mathrm{~B}$ \\
\hline D6Mit100 & 41.03 & CTTGAGTAGGTCTCAGTGCGG & CACATGCACACACAGAAGCA & 84 & $6-3$ \\
\hline D6Mit36 & 48.93 & ACCATCTGCATGGACTCACA & GTTGAAGAGGACGACCAAGTG & 196 & $6-4$ \\
\hline D6Mit10 & 52.75 & TCAGAGGAACAAAGCAGCAT & CCTGTGGCTAACAGGTAAAA & 200 & 610 \\
\hline D6Mit333 & 60.55 & TCСТCACTACAATTCATCTATTACTGC & TGCTTCTGGTATAGGCAGTTAGG & 122 & 6-333 \\
\hline D6Mit302 & 67.66 & AATGACCCTGGTTAGTGTCAGG & GAATTCCATTCGAGGGGC & 113 & $6-4 \mathrm{C}$ \\
\hline D6Mit14 & 77.64 & ATGCAGAAACATGAGTGGGG & CACAAGGCCTGATGACCTCT & 157 & $6-5$ \\
\hline D7Mit21 & 1.91 & GGGTTGAACCTTACAGGGGT & ATCAAACCAGCCCAAGTGAC & 192 & $7-0 \mathrm{~A}$ \\
\hline D7Mit152 & 2.71 & GCCTAGCACACGCCAAAG & CCTTGTGCATGGTTGCTATG & 129 & 7-152 \\
\hline D7Mit57 & 9.94 & TTCCСТCTAGAACTCTGACCTCC & AGTTCAGAGCCGAGACTAGGC & 148 & 7.-1 \\
\hline D7Mit267 & 17.09 & СTCTTTCTGTTACATGGTTAGATTTCC & AAAGACAGTTGAAGTTGACTTCTGG & 192 & $7-2$ \\
\hline D7Mit158 & 29.82 & CTTCATCTGAGCCTGGGAAG & ACTGTAGACCCATGTTCTGATTAGG & 149 & $7-158$ \\
\hline D7Mit317 & 41.5 & ATGTCTCCTTGACATTGGGC & TCTTGTAATCTCACATCTAAGTGTGTG & 102 & $7-2 \mathrm{D}$ \\
\hline D7Mit194 & 44.83 & GTGCAACACACAGAAAAGTTCC & AAAGGCTCACAACGGACTGT & 146 & 7-194 \\
\hline D7Mit220 & 55.69 & AAGCATGCAAGCACACTCAC & ATGCACACAGGCAGTCACTC & 135 & $7-3 \mathrm{~A}$ \\
\hline D7Mit101 & 69.01 & TACAGTGTGAACATGTAGGGGTG & TCCCAACATGGATGTGCTAA & 111 & $7-4$ \\
\hline D7Mit223 & 88.85 & ATGCACATGAGTGTGTGTATGC & TCCTGTGTCTGACGCTCATC & 106 & $7-5$ \\
\hline D8Mit143 & 11.59 & AGCCTGAGGTTATGTTTTTTGC & GGCCCTCAGGTTTTCTCTCT & 279 & 8-143 \\
\hline D8Mit178 & 34.43 & AAAATCAACTGTTTACATTTGAGCC & AGAGCACGCAGTGTGTATGC & 148 & $8-2$ \\
\hline D8Mit45 & 42.16 & GAACAGGACCAATAAAATGAAAGC & СТАССТTACСAAACTTCCCGG & 121 & $8-45$ \\
\hline D8Mit80 & 43.06 & TGCATTTGTCAGGGCTCTC & ATGACACATGAGCCTCCACA & 107 & 8-080 \\
\hline D8Mit211 & 52 & CAGAACACTGTCCTGAAAAGTCC & TACCCACAAACCTGTATTTAAATTAA & 149 & $8-4$ \\
\hline D8Mit215 & 62.63 & AATACACAAGGTTGGCCTCA & ATGTGTGGATATTCATGTGCTC & 178 & $8-5$ \\
\hline D8Mit56 & 76.14 & ACACTCAGAGACCATGAGTACACC & GAGTTCACTACCCACAAGTCTCC & 162 & $8-56$ \\
\hline D9Mit250 & 2.46 & CCCAAAAACCTATTTGCAGTG & GTGACATGATTCCTTCAGTCTTACC & 123 & $9-1$ \\
\hline D9Mit249 & 2.46 & AAGCCCTCTTAGAAGTAGTGTGTATG & AGCCATGAACTAACTTACATGTATCA & 125 & 9-249 \\
\hline D9Mit89 & 14.79 & CACATACAAGGATATACATACACAGGC & TCACAGGAGGTGGCAGAAAT & 145 & 9-89 \\
\hline D9Mit285 & 21.42 & CAAATACATTGCTGATTATATCAGAGA & GGACTCTAGATCTCATCAGGGA & 125 & $9-2$ \\
\hline D9Mit336 & 35.39 & AAGTGGTTCACAGAAATGTATACAGG & TTTTCTTTCTGTGGTAAAGGGG & 122 & $9-3$ \\
\hline D9Mit8 & 42.65 & GATGAAGACAATAAAGAACCTTAAA & AAGAGCTAACCCATTGCTGC & 183 & $9-3 B$ \\
\hline D9Mit347 & 55.11 & ССТCCACATGTGCACTGCT & CTGTCCATCTATCATCTATCTGTCTG & 122 & $9-4$ \\
\hline D9Mit18 & 71.49 & TCACTGTAGCCCAGAGCAGT & CCTGTTGTCAACACCTGATG & 180 & $9-5$ \\
\hline D10Mit28 & 3.04 & CCTCCTGTATGTGTATTTAAAGCA & CTGCCCATCTGACCCTGATA & 147 & $10-1 \mathrm{~A}$ \\
\hline D10Mit213 & 9.75 & СТССТССТACTGATTGTCCCC & GGGACAAACTTTTAAAAATTGCA & 150 & $10-2$ \\
\hline D10Mit108 & 22.89 & TGCCTGTAACCTGCATACCC & GTTTAACACCCAGGACTATACATGG & 142 & $10-108$ \\
\hline D10Mit20 & 34.83 & CACCCTCACACAGATATGCG & GCATTGGGAAGTCCATGAGT & 234 & $10-3$ \\
\hline D10Mit230 & 45.28 & AGATAGCCTAGGGGGTGCAT & ATCAGTTTCCAATCGCTGCT & 115 & $10-4$ \\
\hline D10Mit96 & 51.19 & CTTCTTTGAAGTTAGATGCAGCC & TACGGAGAAGGGAACACCTG & 150 & $10-096$ \\
\hline
\end{tabular}


TABLE 2: Continued.

\begin{tabular}{|c|c|c|c|c|c|}
\hline Marker & $\mathrm{cM}$ & Primer $\mathrm{F}$ & Primer R & Product size & Lab code \\
\hline D10Mit178 & 51.42 & ATTGTCAAATATCTTCCTCAGTTGC & TTATTCCTAGGCAGTCTGTCTGG & 133 & $10-4 \mathrm{~B}$ \\
\hline D10Mit233 & 61.58 & GTGCTTTATATTGGAGATCATCACA & GTCCCGAATTTCACATACATAGC & 130 & $10-5$ \\
\hline D10Mit271 & 72.31 & ACAACCAAAGGTCTTTGTAGAAGA & AATATATAGGCACACCTTAATAGCCA & 117 & $10-6$ \\
\hline D11Mit1 & 2.51 & GGGTCTCTGAAGGCTTTGTG & TGAATACAGAAGCCACGGTG & 153 & $11-1 \mathrm{P}$ \\
\hline D11Mit226 & 5.64 & AGGTGAACTCTTTTGAAGTTTGTG & AAAGGAGTGACTGAGAAAGACACC & 139 & $11-226$ \\
\hline D11Mit151 & 15.29 & TGGGAATTCTGGGAGTTCTG & GTTGGTCTGTTATGAAGACCAGG & 140 & $11-1 \mathrm{Q}$ \\
\hline D11Mit217 & 23.1 & ACTGGAAAATATGTTTTAAACCTCTG & AAATGGGATTCTGCAAAAACC & 135 & $11-217$ \\
\hline D11Mit349 & 33.29 & AGTATCAGAAGATCCAGTTGGAGG & GTAGAAAAAGATACCCAGTGTCAGC & 118 & $11-349$ \\
\hline D11Mit298 & 42.76 & AAACAAACAAAAATGCACCTCA & GTACCACCATGCCTAGCCTC & 199 & $11-298$ \\
\hline D11Mit39 & 52.22 & TTTCATGACCCCTAATTTCCC & GTGGGTGTGCCTGTCAATC & 155 & $11-39$ \\
\hline D11Mit70 & 58.9 & GGAAGTAGCTATGGAGGTGGC & TCTGACCCAGAGCTCAAATACA & 140 & $11-70$ \\
\hline D11Mit54 & 59.82 & AGGCTGGTGGCTAGTGTCC & AAGTCTTGCGCTGCATCTTT & 144 & $11-4 \mathrm{~A}$ \\
\hline D11Mit132 & 62.92 & GGTCAGAGGACAATCTTACATGC & GTTCCAAGACAATGAGAGACCC & 117 & $11-4 \mathrm{~B}$ \\
\hline D11Mit333 & 71.83 & CATGTGGTTATTTTCTAGCCCC & AGGCATCAATAACTATTTTTCAGTG & 125 & $11-5$ \\
\hline D11Mit303 & 82.9 & TCAATCTCTCAAGTTTTTCCAGC & GACAACGTTGACCTCCACG & 106 & $11-6$ \\
\hline D12Mit12 & 8.49 & TTCAATGCCTTCTGGCTTCT & GATTACCGGGTGTGTGACCT & 145 & $12-1$ \\
\hline D12Mit2 & 18.94 & ACACAGGCTAAAACATGGGC & GCATCTGTATTCCACAGGCA & 134 & $12-2$ \\
\hline D12Mit114 & 28.94 & TTGACCTTGAACTTGTGACCC & GTTTTCTCCAAATCACTGTCACC & 144 & $12-3$ \\
\hline D12Mit149 & 36.85 & CATGGCACACATACATACATGTG & AACATAGCAATGGTATATAGGTATGGG & 132 & $12-3 \mathrm{~B}$ \\
\hline D12Mit117 & 43.32 & AATTGAGGAACTTAGAAGAAAAGCC & CCTCTGGCCACCATACATG & 127 & $12-117$ \\
\hline D12Mit30 & 50.43 & TATGTGACTGCAATCCCAGC & ATGAACACATCATGCCCAGA & 107 & $12-30$ \\
\hline D12Mit99 & 52.9 & CTTACAGAAAATGAAAACCAAAACA & CCTCTGCTTTAGAGGCAAACG & 151 & $12-099$ \\
\hline D12Mit263 & 62.11 & TCAGATCTCAGCAGATAAATACTTGG & TCCCCTGGAGCATATTTGAC & 113 & $12-6$ \\
\hline D12nDs2 & 62.22 & ACATGGTAATTTATGGGCAA & CTGGATACCTGCAATAGTAGA & 195 & $12-5$ \\
\hline D13Mit16 & 7.26 & CCAGCTGAAGGCTTACTCGT & AAAGTTAGAATCAGCCATTCAAGG & 207 & $13-1$ \\
\hline D13Mit137 & 18.87 & GAATCAGAGAACCTGGCTGTG & TCTAAAAGAGAGAAAATTGGGGC & 131 & $13-137$ \\
\hline D13Mit63 & 21 & GAGATGGAAGGAAGAGATGGC & CAAATGCATGTATCCGTATGTG & 139 & $13-63$ \\
\hline D13Mit186 & 31.87 & GAAAGCCCTAGGGGAAGATG & TGCAGTTTCTAAGGTTAAAACTAAAGC & 149 & $13-3$ \\
\hline D13Mit282 & 32.59 & TCGCACTTCCTATACAGTTATAAGAG & GGACAGAAAGCATGCAGAGG & 125 & $13-282$ \\
\hline D13Mit191 & 45.05 & GCAAATTAGAGAATCATGCATCC & TGGAGAATGCTAAAAGCATGG & 119 & 13-3B \\
\hline D13Mit51 & 56.45 & TCCTGCAAAAGTGGAGCC & TGGAAACAAGCTCTTGGAGG & 146 & $13-3 \mathrm{P}$ \\
\hline D13Mit213 & 59.69 & GCCTGAAACTCTACATAAAATACATCC & AGTTTCATTGCTTTAGTTACATTTTCA & 149 & $13-5$ \\
\hline D13Mit78 & 67.21 & ACAGCACGGGTTTATCATCC & TATGCCTGCCAGGCTTCTAT & 229 & $13-6$ \\
\hline D14Mit179 & 4.92 & CCACTTGCAGCATTGACAAT & CAAACATCTGTGACAATAAAATTTCA & 144 & $14-1 \mathrm{P}$ \\
\hline D14Mit126 & 11.94 & CCTGTCCCACAACACCTTTT & TATACATATGGGTAGCACTGAGTGG & 140 & $14-2$ \\
\hline D14Mit60 & 24.6 & AGGCTGCCCATAAAAGGG & GTTTGTGCTAATGTTCTCATCTGG & 132 & $14-3$ \\
\hline D14Mit259 & 27.65 & TGGTGTCTCCTTCGGAATTT & TAAATGTAAAAGGTAAAGGCAATGG & 125 & $14-259$ \\
\hline D14Mit39 & 35.69 & AAAGAGCAACCCCCAATTCT & ACTTTTACCTGGTCTCCAAAAGC & 246 & $14-39$ \\
\hline D14Mit68 & 37.61 & GTGGCATGCACAACCGTATA & CCCTTTTGAGGTGCTTGTTT & 153 & $14-4$ \\
\hline D14Mit194 & 45.96 & AATATTCTAAATGAAATCCAATGTGTG & TTAATTGCAAGTAACACAATGAGTAGG & 92 & $14-4 \mathrm{~B}$ \\
\hline D14Mit95 & 57.2 & TATTTTTAAGTCAGTATACACATGCGC & TTATCCAAGTGTATTTAAAGAAGAGGC & 124 & $14-5$ \\
\hline D14Mit97 & 62.2 & TCAGTCCAAACTCTGTTAATCTTCC & CAGCTCCACATTTTTGCTCA & 136 & $14-6 \mathrm{~A}$ \\
\hline D15Mit13 & 1.84 & GGAGACAAAAATGAACTCCTGG & TTGTAAGACAAGCATAGCTCAACA & 136 & $15-13$ \\
\hline D15Mit265 & 6.08 & ACATTAGTCAACTATGCTGGTACTCTG & TTCСТCTCTAATGTCAATTGTTTCA & 198 & $15-265$ \\
\hline D15Mit138 & 15.68 & TTCAATTCCCTTTTGTCAAATG & CAAGACCCTAGATTCAGTCTACCC & 147 & $15-138$ \\
\hline D15Mit154 & 16.82 & AGCACTGGGTACACAAACTGG & ATGAAAGCATGTGTAGTCTTTCTCA & 150 & $15-2$ \\
\hline D15Mit128 & 25.58 & CAAGTTCTGCAAAGAATTATTTATGC & CCACTTTGAAGTTTTCTTTCTTAGC & 134 & $15-3$ \\
\hline D15Mit92 & 32.19 & AGTCTCTCTCССССТTСТCTC & TGCCACAAGCACAATAGTATCC & 147 & $15-3 \mathrm{~B}$ \\
\hline D15Mit80 & 38.02 & CATTGAGGGTTTGTAGGTTGG & ACCCCTGCAAGTTGTCTTTG & 149 & $15-4$ \\
\hline D15Mit34 & 45.31 & TGGACAACCATTTTGGACAA & CTTTCTGTCAGGCATCACCA & 147 & $15-34$ \\
\hline
\end{tabular}


TABLE 2: Continued.

\begin{tabular}{|c|c|c|c|c|c|}
\hline Marker & $\mathrm{cM}$ & Primer $\mathrm{F}$ & Primer R & Product size & Lab code \\
\hline D15Mit244 & 48.65 & TCTACCCTCTGTGGAACATCG & CTTTGTGTCCATACACTAATATCAAGG & 116 & $15-4 \mathrm{P}$ \\
\hline D15Mit16 & 58.05 & AGACTCAGAGGGCAAAATAAAGC & TCGGCTTTTGTCTGTCTGTC & 119 & $15-6$ \\
\hline D16Mit131 & 3.41 & TGGTGGTGGTGTTGATGGTA & AAGACCATTTCTAATAAACAACACCC & 140 & $16-1$ \\
\hline D16Mit136 & 27.82 & AGATAATTCCCGTGAGAATAAAACC & TTGAGAAGTTTGCCCTATAATGG & 129 & $16-2$ \\
\hline D16Mit30 & 33.01 & GTGCACATACATACCACAGCG & TCACTGCAGGGAGGTTCAG & 152 & $16-30$ \\
\hline D16Mit64 & 34.22 & TACCATGATCAGTCCAAAGGC & ACTTAAGGTTGTCCTGTGGGG & 220 & $16-3$ \\
\hline D16Mit140 & 40.3 & ATAGTTGAAAAACTTGAACATGCG & GAAAAGGTTAATGCTGGTCACC & 145 & $16-3 \mathrm{~B}$ \\
\hline D16Mit19 & 45.36 & CAGGCATGTGAACAAAGTGG & GTGACTGATGAATGCCTGACA & 121 & $16-3 \mathrm{C}$ \\
\hline D16Mit70 & 48.81 & GGATCTATATGCTATAGAACCATTCA & GTCATCAATTCCATTTCCTAATATAGA & 187 & $16-4$ \\
\hline D16Mit71 & 57.06 & TAGAAAATCTTCAAATAGGATCTGTTC & GAGCATTTCCCTTTTACCTGG & 154 & $16-6 \mathrm{~A}$ \\
\hline D17Mit164 & 2.11 & AGGCCCTAACATGTAGCAGG & TATTATTGAGACTGTGGTTGTTGTTG & 133 & $17-1$ \\
\hline D17Mit133 & 12.53 & TCTGCTGTGTTCACAGGTGA & GCCCCTGCTAGATCTGACAG & 188 & $17-1 \mathrm{C}$ \\
\hline D17Mit51 & 19.74 & TCTGCCCTGTAACAGGAGCT & CTTCTGGAATCAGAGGATCCC & 154 & $17-2$ \\
\hline D17Mit68 & 23.55 & GTCCTGACATCATGCTTTGTG & CTACCGTTTGGAAGGCTGAG & 130 & $17-68$ \\
\hline D17Mit20 & 29.73 & AGAACAGGACACCGGACATC & TCATAAGTAGGCACACCAATGC & 165 & $17-3$ \\
\hline D17Mit205 & 39.3 & TGTGCATGTATATGTGTGTGAATG & GCTAAGTCAGAAGAGTTCTGTAATGG & 223 & $17-4$ \\
\hline D17Mit1002 & 50.97 & TCTGAATGCTGACTTCCATCC & ACACATATGTGTAGTGTATGAATGTGC & 138 & $17-4 \mathrm{P}$ \\
\hline D17Mit123 & 60.67 & CACAAGGAGGGAGCCTGTAG & CACCGTAAGAGTCTAATAATAAGGGG & 133 & $17-6$ \\
\hline D18Mit222 & 8.08 & AATCCAAGATTGACATGTGGC & CTTAGATGCCCTGTCTTAAAAAAA & 113 & $18-1$ \\
\hline D18Mit226 & 18.18 & CAGGCAGGGTGCATATATTATAA & TATCTGTTTATGTGTGTACATTGTGTG & 125 & $18-1 \mathrm{C}$ \\
\hline D18Mit177 & 21.39 & CTGTAGTTTATCAGTTCACCCTGTG & TGTGCTGTTAAACAAATATCTCTGG & 171 & $18-2$ \\
\hline D18Mit91 & 29.67 & TCCACAAATGTTGGCAAAGA & TTTCTGGCCATATTGGAAGC & 140 & $18-3$ \\
\hline D18Mit124 & 32.15 & CCCAAATGGGGTGTCTTTTA & CTGCCACACATTTGTGTGTATG & 150 & $18-4$ \\
\hline D18Mit184 & 39.7 & CACACATGTGTAGGTAGGTAGGTAGG & CGCACAAGGACTACTGAAACA & 172 & $18-4 \mathrm{C}$ \\
\hline D18Mit186 & 45.63 & AAGTGTTGGGCAAAGGCTAA & CTTTAGTATAGTGTGCATGAGTGTGA & 125 & $18-5$ \\
\hline D18Mit7 & 51.92 & ACAGGAGAACGGGAACTCAG & GCCAGAGTGGACCAAGATGA & 95 & $18-5 \mathrm{~B}$ \\
\hline D18Mit129 & 53.28 & CCAGCACAGAGGCAGTCAT & TGATTCTTGGGTCCTGAATACA & 138 & $18-5 \mathrm{C}$ \\
\hline D19Mit68 & 3.38 & CCAATACAAATCAGACTCAATAGTCG & AGGGTCTCCCCATCTTCCTA & 132 & $19-1$ \\
\hline D19Mit60 & 13.9 & CAACACCTCACTGTTTAGGAACC & GCTGAGGTCAATATTTAGCATGC & 136 & $19-60$ \\
\hline D19Mit45 & 16.14 & CCATTCATAAAATGGGCTTAGG & ACCATGAATGTGTTTTGAGGTG & 145 & $19-2 \mathrm{~A}$ \\
\hline D19Mit30 & 21.34 & GGTGGCTTAGAAATAGTATCGAAA & CCAGCTCTAGGCAGGCATAT & 150 & $19-2 \mathrm{~B}$ \\
\hline D19Mit88 & 32.23 & AACAGTGCAACTTTGGAGGC & TCATTGGAACTGTCTTAACAGTGC & 148 & $19-3$ \\
\hline D19Mit19 & 34.08 & CCTGTGTCCATACAGGCTCA & ACCATATCAGGAAGCACCATG & 138 & 19-19 \\
\hline D19Mit11 & 36.26 & TCAAAGTCAAGGTGGGCAG & ACTTTCCAGATGTTGGGCAC & 146 & $19-4$ \\
\hline D19Mitl & 50.32 & AATCCTTGTTCACTCTATCAAGGC & CATGAAGAGTCCAGTAGAAACCTC & 122 & $19-5$ \\
\hline D19Mit71 & 56.28 & ATGATTCCCGCAGTTTTGTC & TCTCAACTGTTATTCCTCAATAGCC & 136 & $19-6$ \\
\hline DXMit136 & 4.23 & ACGGAAACACTCTTATGTGCG & ATTTTGATTACAGCATGTCCCC & 182 & $\mathrm{X}-136$ \\
\hline DXMit48 & 25.51 & АССТСССССТGСАТТАСТСТ & TTCTCCAGAATCCATGCTCC & 105 & $\mathrm{X}-48$ \\
\hline DXMit62 & 34.6 & GCAATTGTGATGTTGTAGTAAATATGG & ATAACTGAGGTCTGCGGGG & 125 & $\mathrm{X}-62$ \\
\hline DXMit110 & 35.53 & TGACATGAAGTATGTGTTCCTGC & TAGGCACATGTTCACATGGG & 135 & $\mathrm{X}-110$ \\
\hline DXMit170 & 45.87 & TGCAGGCACTAACAGTGAGG & TAGTTTCACTGTGCCATTGTATACA & 115 & $\mathrm{X}-170$ \\
\hline DXMit179 & 53.17 & TTTGATAGAGCCATGTTTGGC & CAGGCTAGCCTCAAACTCTCA & 122 & 73.26 \\
\hline DXMit79 & 68.46 & AGTCTGCCTTCTCTTTCTGTATCC & TGAAACTATTCCAACATTATTCTTGG & 138 & 53.17 \\
\hline DXMit153 & 73.26 & CAATCAAGCAGATGGAAGCA & AAGGACTGCCAAGAGGACAA & 143 & 68.46 \\
\hline
\end{tabular}


TABle 3: The markers that yielded PCR bands but could not be included in the panel due to their not meeting the set criteria.

(a) Category 1: markers that yielded the same size bands in all the strains

\begin{tabular}{lcllc}
\hline Marker & cM & Primer F & Primer R & Lab code \\
\hline D2Mit74 & 103.43 & CCAAGCTTGCAGTTTGTTAGC & AGGTGTTATTGAGCCCTGTATAGC & 2-6A \\
D10Mit88 & 28.64 & AAGATGAGAAGATAACATGTCAGGC & TTCTGAATTAAGTTCATCTGAACCC & 10-2C \\
D11Mit226 & 5.64 & AGGTGAACTCTTTTGAAGTTTGTG & AAAGGAGTGACTGAGAAAGACACC & 11-1 \\
D11Mit60 & 42.86 & AGAGAGGCAAAAATTCCAAGC & CTTCCTGATGGTAGGATTTAGGC & 11-60 \\
D16Mit51 & 53.78 & CCTCAGGTCAGTCAGGATTTAA & CCTGTTCACCCTCTCCACAT & $16-5$ \\
DXMit57 & 16.24 & AGTAGCAAGTAGACTCTCAAAGAGGG & TCTGGCATACATGGGCACT & X-57 \\
DXMit81 & 20.59 & GAGGAGCATCAACCTTCTCG & GAGGTGGGGAGAAACAGAGG & X-81 \\
DXMit63 & 41.51 & TTATAAATTAGTGTTACCACATGCAGC & AACATTTTTTTCCTAGCATGTGTG & X-63 \\
DXMit186 & 76.75 & ATCAATGCATAGTATTTGGGCC & AATTTGTCACTGCGGGTAGG & X-186 \\
\hline
\end{tabular}

(b) Category 2: markers that failed to amplify a PCR band in some strains

\begin{tabular}{|c|c|c|c|c|}
\hline Marker & $\mathrm{cM}$ & Primer F & Primer R & Lab code \\
\hline D1Mit375 & 23.18 & TAAATCCATAGATGATAGATCAGTGTG & GTGGAAAAAAAACCTAAGACACC & $1-2 \mathrm{C}$ \\
\hline D1Mit76 & 33.31 & ACAAAGGAAACTAAACAGACTCGG & СТСССТСAAATACATCTTTGGC & $1-3 \mathrm{C}$ \\
\hline D1Mit308 & 50.78 & GAGGCTATGAGTCAAATGGACC & TTTATGAGGTGCTGAGATGCA & $1-5$ \\
\hline D2Mit448 & 65.66 & TACTGTTTGCATTTGAGTGCG & AAAAAGTAATGGTTGGGGCC & $2-4 \mathrm{~B}$ \\
\hline D3Mit278 & 32.59 & TCTAATATTGGAAAATGAATTTCTCTC & TATGCCCACATGCACACC & $3-3 \mathrm{P}$ \\
\hline D5Mit309 & 42.22 & TAGAGCCTATTTCAAACCCCC & GTTGCATCCATAGCAAGCAA & $5-4$ \\
\hline D10Mit49 & 1.91 & GGAATTTACACTGGAATACAACCC & GTGGGCATTTGCACTGTG & $10-1$ \\
\hline D11Mit168 & 78.74 & CAGGGATTTGACTTTTAACCTCC & GAAATGGCTCCTACAACСТCC & $11-168$ \\
\hline D14Mit132 & 6.03 & GAACAGCACCATCCACACAC & GTGGGGTTATATGCAGATACTCG & $14-1$ \\
\hline D15Mit147 & 46.85 & GATGTGTGAAAAATTTTGTTTCTTG & GTCTCAAAGGAATAAGAAAGAGATGG & $15-5$ \\
\hline D17Mit3 & 34.9 & GATCTTTTCTTATTCTGGTT & GCAAAGTCATGTACTCTGAG & $17-3 \mathrm{~B}$ \\
\hline D17Mit39 & 45.64 & CCTCTGAGGAGTAACCAAGCC & CACAGAGTTCTACСTCСAACСC & $17-5$ \\
\hline D18Mit48 & 51.89 & TTGCACTCACAGGGCACAT & TCAGAGTTTCCAGAAGACACCA & $18-6$ \\
\hline D18Mit25 & 59.05 & CTGGAAATAAAACCTGGGCA & TTTAGCCTAACTGAGTTCCAGACC & $18-7$ \\
\hline D19Mit31 & 8.84 & CAATACAGAGTAATGATTGCCTGA & TTCACATTTTGGGATGCTCA & $19-1 \mathrm{~B}$ \\
\hline D19Mit41 & 13.18 & AGCCCTCCACCCAGTTTC & TCTGGGGAAAAAGGATGAGA & $19-2$ \\
\hline DXMit124a & 4.25 & AAGGAGAAGTAGAAGAAAGAAAAGAGG & GGTGTAGCCTCAAAAAAGATGG & $\mathrm{X}-124 \mathrm{a}$ \\
\hline DXMit68 & 29.49 & TCCTTTGGCCTCCTGCATAT & TGTTCTTACAATGAGCCTCATAGG & $\mathrm{X}-68$ \\
\hline DXMit114 & 42.82 & ATGGCATCCACAGTACCACA & GTAAAATCAATTTGTGAATAAGGAAGC & $\mathrm{X}-114$ \\
\hline DXMit95 & 45.86 & CTGTCAATCTAATTCTCTATGTCTGTG & СTTTCCTGGGTGGCAGTG & $\mathrm{X}-95$ \\
\hline DXMit155 & 67.49 & TGTGCACCACCACCATTC & AGGATCTGAGTGCCCAACC & $\mathrm{X}-155$ \\
\hline
\end{tabular}

(c) Category 3: markers that amplified multiple PCR products in most (or all) strains

\begin{tabular}{lcllc}
\hline Marker & cM & Primer F & Primer R & Lab code \\
\hline D11Mit86 & 32.13 & TTGACATTGTGACAAAGACTTTCA & AAGGCATCATGAGGTTTTTAGTG & $11-3$ \\
D12Mit118 & 44.93 & CATCTTCAATAAAATGGAGATGTACA & CGCTTTCCCTTCATGTACTAGC & $12-4$ \\
D13Mit107 & 50.2 & CAACTGAGCCACATTCCTAGC & CAGCCACAGGATGATGAGG & $13-4$ \\
DXMit98 & 72.38 & GAGAGCAGGACTATGACTTG & ACACACCAGTTCGCACACAT & X-98 \\
DXMit222 & 78.31 & TTGGTTTGGGGTTTTTTTTG & ATTCCTGATAATGTCTTCTGGACA & X-222 \\
\hline
\end{tabular}

not yield a reliable PCR product in most/all of the strains) might have not worked due to technical errors that may have occurred during some steps like primer synthesis or primer reconstitution; we do not rule out the possibility that some of these primers may yield PCR products if they are resynthesized. The markers that did not meet our criteria because of the above reasons are listed in Table 3 and Supplementary Table S1: providing the list of primers and markers that failed in our hands will help researchers to compare the results if they intend to test more markers to expand the streamlined panel presented here.

There is some debate about the minimum number of markers needed per chromosome for identifying the regions between the donor and recipient strains [32]. A study concluded that as few as three markers per chromosome were sufficient to achieve similarly meaningful results as that of 
over 6 markers per chromosome [33]. Computer simulation [20] indicated that a relatively modest selection effort of 60 evenly spaced markers with $25 \mathrm{cM}$ spacing (corresponding to 3 markers per chromosome), 16 males per generation, would typically reduce unlinked donor genome contamination to below $1 \%$ by four backcross generations (N5). We conclude that the list presented here can serve to choose panels of markers for most two-strain combinations with at least 3 to 4 markers per chromosome (with exception of a very few combinations). Further studies that compare smaller and larger panels of markers in the same set of samples for marker assisted speed congenics are needed to address this question unequivocally.

In recent years there have been advancements in the approaches used for speed congenics. These methods include (i) use of fluorescently labeled primers to amplify SSLP markers followed by resolving the products in sequencing gels [29] and (ii) microarray chips of SNPs [29, 34-36]. With the advent of newer methods particularly those that use SNP based marker analysis, a very high number of markers per chromosome can be screened simultaneously that increases the resolution severalfold compared to the conventional SSLP based markers analysis. Although there are some computer simulation studies for assessing the efficiency of speed congenic screening in general [32] and algorithm based reports to compare SNP and SSLP based speed congenic screens [37] there are no systematic studies to compare the two methods to assess the efficiency and cost effectiveness of each method. Here, we compare the SNP and SSLP based approaches in terms of their adoptability and feasibility to most laboratory settings including their cost. The hands-on-time in performing gel based assays has been reduced greatly by newer methods that use SNP arrays. However these methods have some limitations compared to traditional agarose gel electrophoresis (AGE) based SSLP marker analysis. (1) The newer methods are expensive in terms of the initial investment in reagents and/or operational costs compared to SSLP-AGE method. On the contrary, basic requirements needed for AGE based systems are readily available in any molecular biology laboratory and the only additional investment needed will be to synthesize the required oligonucleotides. (2) Subset of markers to be analyzed in the subsequent generations cannot be skipped from the panel unlike in the AGE based method where the number of markers to be analyzed will become significantly reduced in successive generations and so also the overall cost of the assay. Other advantages of AGE based SSLP marker analysis over these newer methods are as follows: (i) the equipment needed to perform the assay is readily available in most laboratories and (ii) it can be routinely performed by many researchers and technicians without the need of special training as needed for SNP based approaches.

Considering the highest resolution that is possible with the SNP based method, it can be regarded as the superior method of all. However, the microchips that are currently available are expensive and the cost for analyzing each mouse DNA sample runs to about US $\$ 100$ to $\$ 150$. Assuming that about 15 mouse DNA samples per generation for 5 generations are analyzed, a typical speed congenic project would cost about $\$ 15,000$ to $\$ 22,500$. On the other hand, when using
SSLP-AGE approach, since marker analysis is done manually, the markers that were fixed in the previous generation can be skipped in the successive generations; this makes the SSLP-AGE based method cost effective compared to SNP based method. It is estimated that a typical SSLP based speed congenic screen that employs analysis of 15 samples per generation for 5 generations using 5 markers per chromosome would need about 2000 to 2300 PCR reactions. At the rate of $\$ 2$ to $\$ 2.5$ per reaction (cost analysis done in our laboratory) it will cost about $\$ 4000$ to $\$ 5750$ for one full speed congenic project. Furthermore, SSLP-AGE method can be performed in any simple molecular biology labs compared to SNP method that requires expensive equipment.

\section{Conclusions}

Although some information about microsatellite marker differences between the commonly used inbred mouse strains was available, there was no systematic study to validate a large panel of markers for SSLP-AGE based speed congenic screening. The panel of marker sets validated and presented in this study serves as a ready reference for researchers who wish to perform cost-effective speed congenic screening in a pair of strains from the panel. The assay can be performed in any standard molecular biology lab. The data in this report is available at $\mathrm{ftp}$ ://ftp.informatics.jax.org/pub/datasets/ index.html\#Guru.

\section{Conflict of Interests}

The authors declare that there is no conflict of interests regarding the publication of this paper.

\section{Authors' Contribution}

Channabasavaiah B. Gurumurthy and Poonam S. Joshi contributed equally. Channabasavaiah B. Gurumurthy designed the study. Poonam S. Joshi, Scott G. Kurz, Rolen M. Quadros, and Donald W. Harms performed experiments. Channabasavaiah B. Gurumurthy and Poonam S. Joshi compiled the data. Channabasavaiah B. Gurumurthy, Poonam S. Joshi, Masato Ohtsuka, and K. C. Kent Lloyd analyzed and interpreted the data. Channabasavaiah B. Gurumurthy and Masato Ohtsuka wrote the paper and all authors read, edited, and approved the final paper.

\section{Acknowledgments}

The authors acknowledge the review and comments received from Lluis Montoliu. This work was partially supported by an Institutional Development Award (IDeA) to Channabasavaiah B. Gurumurthy (PI: Shelley Smith) from the National Institute of General Medical Sciences of the National Institutes of Health under Grant no. P20GM103471 and the Center for Humanized Mice Development Award from ORIP/DPCPSI/NIH/1R24OD018546-01 (MPI: Gorantla, S/Poluektova, LY). Rolen M. Quadros, Donald W. Harms, and Channabasavaiah B. Gurumurthy acknowledge the Nebraska 
Research Initiative and UNMC Vice-Chancellor for Research Office for supporting the mouse genome engineering core facility.

\section{References}

[1] Y. Cao, X. Liu, N. Deng et al., "Congenic mice provide evidence for a genetic locus that modulates spontaneous arthritis caused by deficiency of IL-1RA," PLoS ONE, vol. 8, no. 6, Article ID e68158, 2013.

[2] S. A. Davie, J. E. Maglione, C. K. Manner et al., "Effects of $\mathrm{FVB} / \mathrm{NJ}$ and $\mathrm{C} 57 \mathrm{Bl} / 6 \mathrm{~J}$ strain backgrounds on mammary tumor phenotype in inducible nitric oxide synthase deficient mice," Transgenic Research, vol. 16, no. 2, pp. 193-201, 2007.

[3] T. Doetschman, "Influence of genetic background on genetically engineered mouse phenotypes," Methods in Molecular Biology, vol. 530, pp. 423-433, 2009.

[4] R. E. Fleming, C. C. Holden, S. Tomatsu et al., "Mouse strain differences determine severity of iron accumulation in Hfe knockout model of hereditary hemochromatosis," Proceedings of the National Academy of Sciences of the United States of America, vol. 98, no. 5, pp. 2707-2711, 2001.

[5] G. D. Gale, R. D. Yazdi, A. H. Khan, A. J. Lusis, R. C. Davis, and D. J. Smith, "A genome-wide panel of congenic mice reveals widespread epistasis of behavior quantitative trait loci," Molecular Psychiatry, vol. 14, no. 6, pp. 631-645, 2009.

[6] T. D. Heiman-Patterson, R. B. Sher, E. A. Blankenhorn et al., "Effect of genetic background on phenotype variability in transgenic mouse models of amyotrophic lateral sclerosis: a window of opportunity in the search for genetic modifiers," Amyotrophic Lateral Sclerosis, vol. 12, no. 2, pp. 79-86, 2011.

[7] K. R. Johnson, Q. Y. Zheng, and K. Noben-Trauth, "Strain background effects and genetic modifiers of hearing in mice," Brain Research, vol. 1091, no. 1, pp. 79-88, 2006.

[8] M. B. Maclennan, B. M. Anderson, and D. W. L. Ma, "Differential mammary gland development in FVB and $\mathrm{C} 57 \mathrm{Bl} / 6$ mice: implications for breast cancer research," Nutrients, vol. 3, no. 11, pp. 929-936, 2011.

[9] X. Montagutelli, "Effect of the genetic background on the phenotype of mouse mutations," Journal of the American Society of Nephrology, vol. 11, no. 16, pp. S101-S105, 2000.

[10] J. Puccini, L. Dorstyn, and S. Kumar, "Genetic background and tumour susceptibility in mouse models," Cell Death and Differentiation, vol. 20, no. 7, p. 964, 2013.

[11] J. A. Siuciak, S. A. McCarthy, D. S. Chapin, A. N. Martin, J. F. Harms, and C. J. Schmidt, "Behavioral characterization of mice deficient in the phosphodiesterase-10A (PDE10A) enzyme on a C57/Bl6N congenic background," Neuropharmacology, vol. 54, no. 2, pp. 417-427, 2008.

[12] S. Thifault, R. Lalonde, N. Sanon, and P. Hamet, "Comparisons between $\mathrm{C} 57 \mathrm{BL} / 6 \mathrm{~J}$ and $\mathrm{A} / \mathrm{J}$ mice in motor activity and coordination, hole-poking, and spatial learning," Brain Research Bulletin, vol. 58, no. 2, pp. 213-218, 2002.

[13] A. B. Auerbach, R. Norinsky, W. Ho et al., "Strain-dependent differences in the efficiency of transgenic mouse production," Transgenic Research, vol. 12, no. 1, pp. 59-69, 2003.

[14] S. Glaser, K. Anastassiadis, and A. F. Stewart, "Current issues in mouse genome engineering," Nature Genetics, vol. 37, no. 11, pp. 1187-1193, 2005.

[15] D. R. Beier, "New genetic resources for mammalian developmental biologists," F1000 Biology Reports, vol. 2, no. 1, article 72, 2010.
[16] M. Inoue, Y. Sakuraba, H. Motegi et al., "A series of maturity onset diabetes of the young, type 2 (MODY2) mouse models generated by a large-scale ENU mutagenesis program," Human Molecular Genetics, vol. 13, no. 11, pp. 1147-1157, 2004.

[17] H. Masuya, K. Shimizu, H. Sezutsu et al., "Enamelin (Enam) is essential for amelogenesis: ENU-induced mouse mutants as models for different clinical subtypes of human amelogenesis imperfecta (AI)," Human Molecular Genetics, vol. 14, no. 5, pp. 575-583, 2005.

[18] C. M. Cendán, J. M. Pujalte, E. Portillo-Salido, L. Montoliu, and J. M. Baeyens, "Formalin-induced pain is reduced in $\sigma_{1}$ receptor knockout mice," European Journal of Pharmacology, vol. 511, no. 1, pp. 73-74, 2005.

[19] P. Markel, P. Shu, C. Ebeling et al., "Theoretical and empirical issues for marker-assisted breeding of congenic mouse strains," Nature Genetics, vol. 17, no. 3, pp. 280-284, 1997.

[20] E. Wakeland, L. Morel, K. Achey, M. Yui, and J. Longmate, "Speed congenics: a classic technique in the fast lane (relatively speaking)," Immunology Today, vol. 18, no. 10, pp. 472-477, 1997.

[21] S. C. Collins, R. H. Wallis, K. Wallace, M. T. Bihoreau, and D. Gauguier, "Marker-assisted congenic screening (MACS): a database tool for the efficient production and characterization of congenic lines," Mammalian Genome, vol. 14, no. 5, pp. 350356, 2003.

[22] S. J. Estill and J. A. Garcia, "A marker assisted selection protocol (MASP) to generate C57BL/6J or 129S6/SvEvTac speed congenic or consomic strains," Genesis, vol. 28, no. 3-4, pp. 164166, 2000.

[23] C. R. Farber, P. M. Corva, and J. F. Medrano, "Genomewide isolation of growth and obesity QTL using mouse speed congenic strains," BMC Genomics, vol. 7, article 102, 2006.

[24] O. A. Iakoubova, C. L. Olsson, K. M. Dains et al., "Microsatellite marker panels for use in high-throughput genotyping of mouse crosses," Physiological Genomics, vol. 2000, no. 3, pp. 145-148, 2000.

[25] N. Ogonuki, K. Inoue, M. Hirose et al., "A high-speed congenic strategy using first-wave male germ cells," PLoS ONE, vol. 4, no. 3, Article ID e4943, 2009.

[26] T. Sakai, I. Miura, S. Yamada-Ishibashi et al., "Update of mouse microsatellite database of Japan (MMDBJ)," Experimental Animals, vol. 53, no. 2, pp. 151-154, 2004.

[27] H. Suemizu, C. Yagihashi, T. Mizushima et al., "Establishing EGFP congenic mice in a NOD/Shi-scid IL2Rg null (NOG) genetic background using a marker-assisted selection protocol (MASP)," Experimental Animals, vol. 57, no. 5, pp. 471-477, 2008.

[28] I. Teppner, B. Aigner, E. Schreiner, M. Müller, and M. Windisch, "Polymorphic microsatellite markers in the outbred CFW and ICR stocks for the generation of speed congenic mice on C57BL/6 background," Laboratory Animals, vol. 38, no. 4, pp. 406-412, 2004.

[29] P. D. Witmer, K. F. Doheny, M. K. Adams et al., "The development of a highly informative mouse Simple Sequence Length Polymorphism (SSLP) marker set and construction of a mouse family tree using parsimony analysis," Genome Research, vol. 13, pp. 485-491, 2003.

[30] C. Lamacchia, G. Palmer, and C. Gabay, "Discrimination of $\mathrm{C} 57 \mathrm{BL} / 6 \mathrm{~J} \mathrm{Rj}$ and 129S2/SvPasCrl inbred mouse strains by use of simple sequence length polymorphisms," Journal of the American Association for Laboratory Animal Science, vol. 46, no. 2, pp. 21-24, 2007. 
[31] I. M. Neuhaus, C. S. Sommardahl, D. K. Johnson, and D. R. Beier, "Microsatellite DNA variants between the FVB/N and $\mathrm{C}_{3} \mathrm{HeB} / \mathrm{FeJLe}$ and $\mathrm{C}_{57} \mathrm{BL} / 6 \mathrm{~J}$ mouse strains," Mammalian Genome, vol. 8, no. 7, pp. 506-509, 1997.

[32] N. J. Armstrong, T. C. Brodnicki, and T. P. Speed, "Mind the gap: analysis of marker-assisted breeding strategies for inbred mouse strains," Mammalian Genome, vol. 17, no. 4, pp. 273-287, 2006.

[33] K. Goto, M. Ebukuro, and T. Itoh, "Microsatellite-directed selection of breeders for the next backcross generation by using a minimal number of loci," Comparative Medicine, vol. 55, no. 1 , pp. 34-36, 2005.

[34] P. M. Petkov, Y. Ding, M. A. Cassell et al., "An efficient SNP system for mouse genome scanning and elucidating strain relationships," Genome Research, vol. 14, no. 9, pp. 1806-1811, 2004.

[35] P. M. Petkov, M. A. Cassell, E. E. Sargent et al., "Development of a SNP genotyping panel for genetic monitoring of the laboratory mouse," Genomics, vol. 83, no. 5, pp. 902-911, 2004.

[36] S. Tsang, Z. Sun, B. Luke et al., "A comprehensive SNP-based genetic analysis of inbred mouse strains," Mammalian Genome, vol. 16, no. 7, pp. 476-480, 2005.

[37] J. D. Gorham, M. S. Ranson, J. C. Smith, B. J. Gorham, and K.-A. Muirhead, " $1+1=3$ : development and validation of a SNPbased algorithm to identify genetic contributions from three distinct inbred mouse strains," Journal of Biomolecular Techniques, vol. 23, no. 4, pp. 136-146, 2012. 

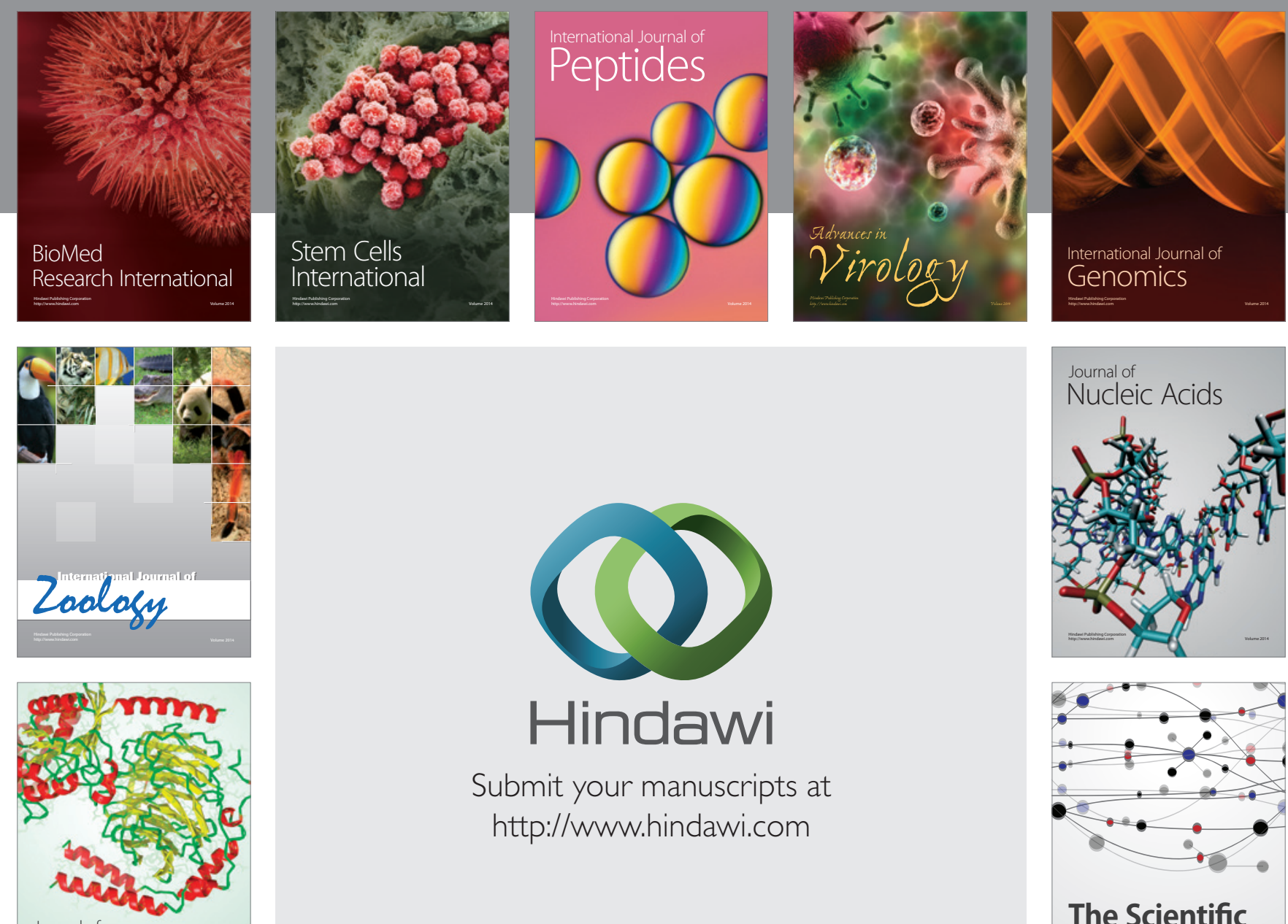

Submit your manuscripts at

http://www.hindawi.com

Journal of
Signal Transduction
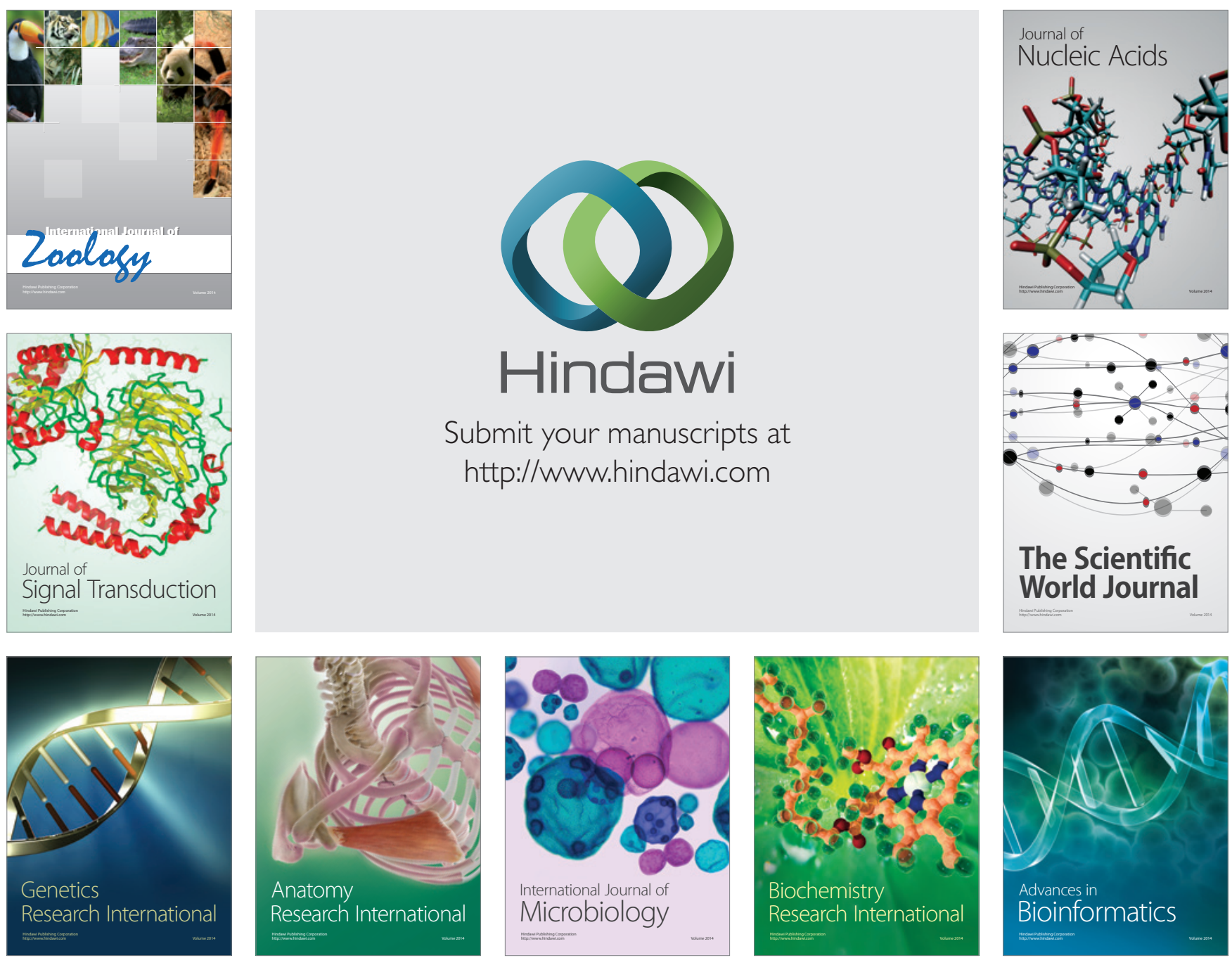

The Scientific World Journal
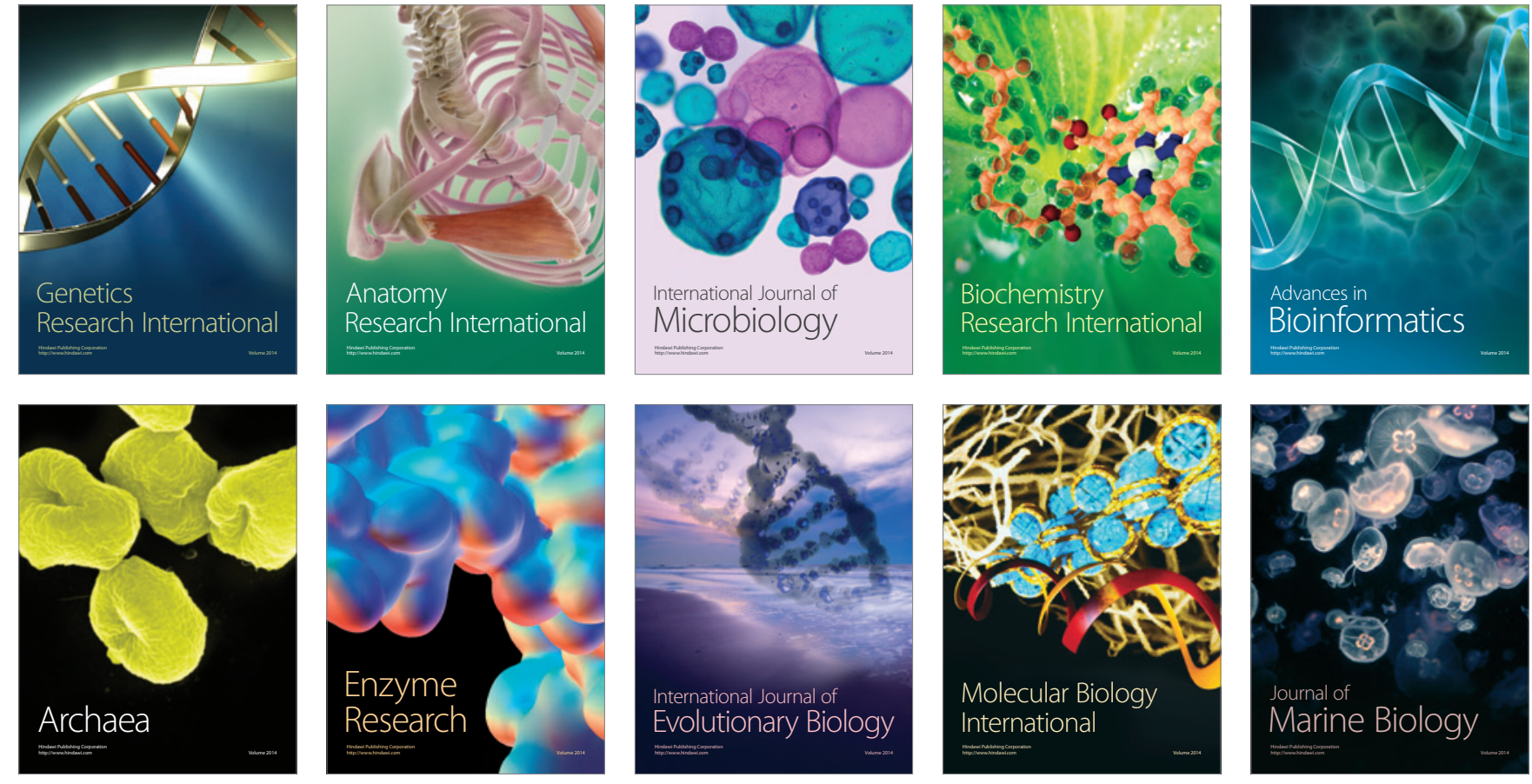